\title{
Natural killer cells activated through NKG2D mediate lung ischemia-reperfusion injury
}

\author{
Daniel R. Calabrese, ${ }^{1,2}$ Emily Aminian, ${ }^{1}$ Benat Mallavia, ${ }^{1}$ Fengchun Liu, ${ }^{1}$ Simon J. Cleary, ${ }^{1}$ Oscar A. Aguilar, ${ }^{3,4}$ Ping Wang, ${ }^{1}$ \\ Jonathan P. Singer, ${ }^{1}$ Steven R. Hays, ${ }^{1}$ Jeffrey A. Golden, ${ }^{1}$ Jasleen Kukreja, ${ }^{5}$ Daniel Dugger, ${ }^{2}$ Mary Nakamura,,${ }^{1,2}$ Lewis L. Lanier, ${ }^{3,4}$ \\ Mark R. Looney, ${ }^{1}$ and John R. Greenland ${ }^{1,2}$ \\ 'Department of Medicine, University of California, San Francisco, California. ${ }^{2}$ Medical Service, Veterans Affairs Health Care System, San Francisco, California. ${ }^{3}$ Department of Microbiology and Immunology, \\ University of California, San Francisco, California. ${ }^{4}$ Parker Institute for Cancer Immunotherapy, San Francisco, California. ${ }^{5}$ Department of Surgery, University of California, San Francisco, California.
}

\begin{abstract}
Pulmonary ischemia-reperfusion injury (IRI) is a clinical syndrome of acute lung injury that occurs after lung transplantation or remote organ ischemia. IRI causes early mortality and has no effective therapies. While NK cells are innate lymphocytes capable of recognizing injured cells, their roles in acute lung injury are incompletely understood. Here, we demonstrated that NK cells were increased in frequency and cytotoxicity in 2 different IRI mouse models. We showed that NK cells trafficked to the lung tissue from peripheral reservoirs and were more mature within lung tissue. Acute lung ischemia-reperfusion injury was blunted in a NK cell-deficient mouse strain but restored with adoptive transfer of NK cells. Mechanistically, NK cell NKC2D receptor ligands were induced on lung endothelial and epithelial cells following IRI, and antibody-mediated NK cell depletion or NKG2D stress receptor blockade abrogated acute lung injury. In human lung tissue, NK cells were increased at sites of ischemia-reperfusion injury and activated NK cells were increased in prospectively collected human bronchoalveolar lavage in subjects with severe IRI. These data support a causal role for recipient peripheral NK cells in pulmonary IRI via NK cell NKG2D receptor ligation. Therapies targeting NK cells may hold promise in acute lung injury.
\end{abstract}

\section{Introduction}

Pulmonary ischemia-reperfusion injury (IRI) is a severe complication following lung transplant that causes primary graft dysfunction (PGD) within 72 hours in one-third of all lung transplant surgeries. Lung transplant is a potentially life-prolonging therapy for patients with end-stage lung disease; however, potential benefits are limited due to complications, which cause some of the highest rates of morbidity and mortality among any solid organ transplant group (1). Early severe IRI, manifesting clinically as primary graft dysfunction (PGD), is a major risk factor for chronic lung allograft dysfunction (CLAD) (2). PGD has no effective therapies and also accounts for nearly $50 \%$ of the mortality observed within the first year after lung transplantation $(3,4)$.

PGD shares clinical features with acute respiratory distress syndrome (ARDS) and is distinct from acute rejection $(5,6)$. Similar to ARDS, it is marked by alveolar-capillary barrier disruption and is diagnosed by bilateral pulmonary opacities and a ratio of arterial oxygen pressure to inspired oxygen content $\left(\mathrm{PaO}_{2} / \mathrm{FiO}_{2}\right)$ less than $300(3,4,7)$. However, distinct from other causes of ARDS, the inflammatory cascade from IRI is thought to play a central pathophysiologic role in $\operatorname{PGD}(8,9)$.

NK cells are innate immune cells that constitute up to $15 \%$ of resident lung lymphocytes (10). NK cells can be activated by the

Conflict of interest: LLL and UCSF have licensed intellectual property rights regarding NKG2D for therapeutic applications.

Copyright: () 2021, American Society for Clinical Investigation.

Submitted: April 9, 2020; Accepted: November 25, 2020; Published: February 1, 2021.

Reference information: J Clin Invest. 2021;131(3):e137047.

https://doi.org/10.1172/JCl137047. recognition of epithelial and endothelial stress molecules (11). IRI leads to HIF-1 $\alpha$ upregulation and induction of these surface cellular stress molecules (12). Upon binding to the NK cell NKG2D receptor, damage signals initiate cytotoxic responses, particularly in mature NK cells (13-16). Outside of the lung, there is evidence that NK cells are important to other IRI responses, such as ischemic cerebrovascular and renal disease, through several mechanisms (13-17).

While other innate immune cells, including macrophages and neutrophils, have been suggested to play a role in the acute lung injury of PGD, the role of NK cells has been incompletely explored. Here, we tested the hypothesis that NK cells mediate pulmonary IRI through recognition of stress ligands in tissue that has been reperfused following ischemia and hypoxia.

\section{Results}

NK cells are increased in the hilar clamp experimental mouse model of pulmonary IRI. To test whether NK cells were increased during pulmonary IRI, C57BL/6 mice were subjected to either hilar clamp (HC) procedure or a sham procedure (Figure 1A). We found that the proportion of $\mathrm{NK}$ cells $\left(\mathrm{CD}^{-} \mathrm{NKp}^{-} 6^{+}\right)$was significantly increased in injured $\mathrm{HC}$ lungs compared with uninjured sham lungs (Figure 1B). Similarly, absolute numbers of NK cells were increased in acutely injured lungs (Figure 1C, $P=0.04$ ). As several other immune cells are capable of similar functions, we also measured $\mathrm{T}$ cells expressing NKp46 (CD3 $\left.{ }^{+} \mathrm{NKp} 46^{+}\right), \mathrm{CD}^{+} \mathrm{T}$ cells $\left(\mathrm{CD}^{+} \mathrm{CD}^{+}\right)$, and myeloid cells $\left(\mathrm{CD} 3^{-} \mathrm{CD} 11 \mathrm{~b}^{+} \mathrm{NKp} 46^{-}\right) . \mathrm{NKp}^{+} 6^{+} \mathrm{T}$ cells were rare but were increased in lungs subjected to $\mathrm{HC}$ by frequency (Figure 1D) and total cell count (Figure 1E). There were no differences in the frequency or count of $\mathrm{CD}^{+} \mathrm{T}$ cells (Figure 1, F 
A Hilar clamp versus sham model

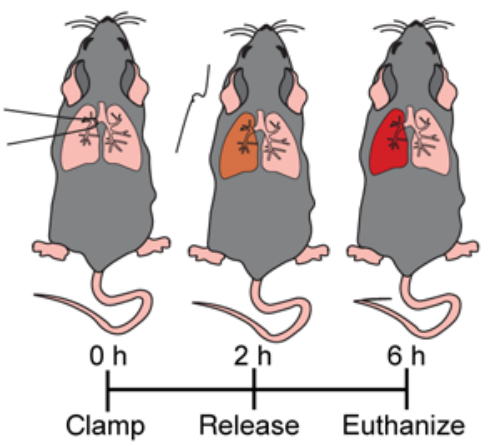

B
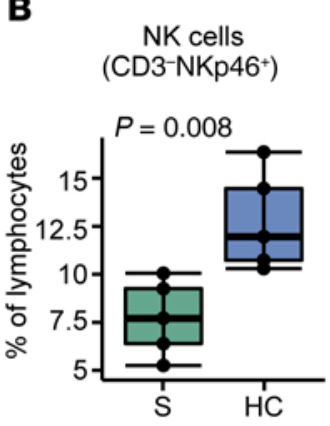

C

$$
\begin{aligned}
& \text { NK cells } \\
& (\text { CD3-NKp46+) }
\end{aligned}
$$

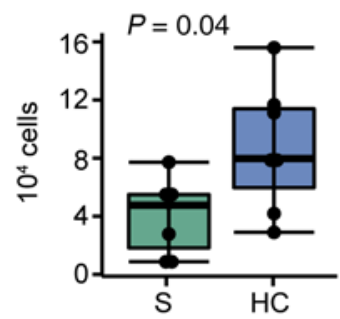

D NKp46+ T cells

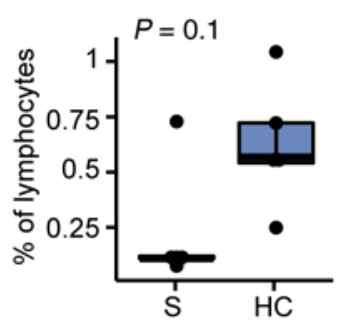

E

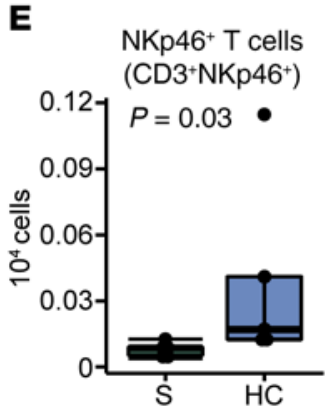

$\mathbf{F}$

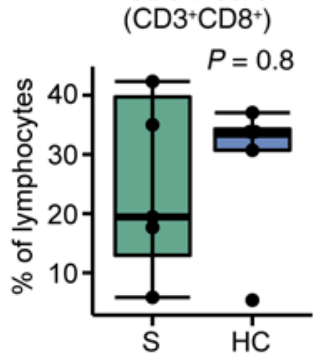

G

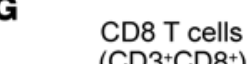

$\left(\mathrm{CD} 3^{+} \mathrm{CD}^{+}\right)$

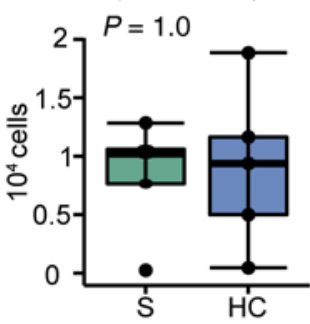

H Myeloid cells
(CD3-NKp46-CD11b+)

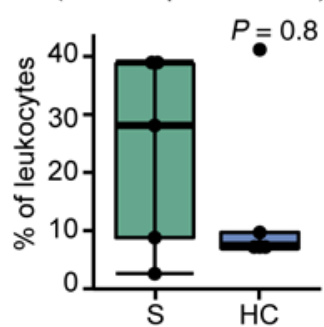

I Myeloid cells
$($ CD3-NKp46-CD11b+)

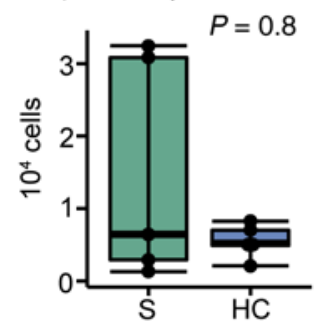

Figure 1. NK cells are increased in injured lungs from a HC model of pulmonary IRI. (A) In the illustrated HC procedure, a slip knot suture is secured around the left hilum and released 2 hours later, followed by 4 hours of in situ reperfusion before mice are euthanized (B). NK cells (CD3-NKp46 $)$ are increased as a percentage of total CD45 cells $(P=0.008)$ and in absolute quantity $(C, P=0.04)$ in dissociated lung tissue following $\mathrm{HC}$ relative to sham procedures $(\mathrm{S})$. CD3 ${ }^{+} \mathrm{NKp} 46^{+} \mathrm{T}$ cells as a frequency $(\mathbf{D}, P=0.1)$ and by total number of cells $(E, P=0.03)$ were increased after $\mathrm{HC}$, but were infrequently identified. CD8 ${ }^{+} \mathrm{T}$ cells as a frequency of total lymphocytes $(\mathbf{F}, P=0.8)$ and in total $(\mathbf{G}, P=1.0)$ were not increased after $\mathrm{HC}$. CD3-NKp46+CD11 b+ cells, which are predominantly myeloid $(\mathbf{H}, P=0.3 ; \mathbf{I}, P=0.8)$, were not different after HC. All experiments studied at least 5 mice for each condition. Box and whisker plots show individual data points bound by boxes at 25 th and 75th percentiles and medians depicted with bisecting lines. Differences were assessed using the Mann-Whitney $U$ test with $P<0.05$ considered significant.

and $\mathrm{G}$ ) or myeloid cells (Figure 1, $\mathrm{H}$ and I) in HC lungs compared with sham lungs. These data suggest that NK cells are the primary early responders in this group of effector cells.

NK cells are more mature and cytotoxic during experimental IRI. With maturation, NK cells gain cytotoxic and effector functions (18). In the mouse, this maturation pattern is characterized by a loss of surface CD27 and gain of surface CD11b in a progression depicted in Figure 2A. To assess differences in NK cell maturation during IRI, we measured CD11b and CD27 surface markers in digested C57BL/6 mouse HC and sham lungs. We found no differences in immature NK cell frequencies between lungs subjected to $\mathrm{HC}$ or sham surgery (Figure 2, B-D). However, we found increased frequencies of mature NK cells $\left(\mathrm{CD} 27^{-} \mathrm{CD} 11 \mathrm{~b}^{+}\right)$in $\mathrm{HC}$ lungs (Figure 2E). Upon cytotoxic NK cell degranulation, lysosomal membrane protein CD107a is increased on the cell surface (Figure 2F) (19). We found increased $\mathrm{CD} 107 \mathrm{a}^{+} \mathrm{NK}$ cells by percentage (Figure 2G, $P=$ 0.002 ) and by MFI on NK cells (Figure $2 \mathrm{H}, P=0.07$ ) from HC lungs compared with those from sham lungs. NKG2D is an activating receptor that recognizes stress molecules and is expressed at steady state on most NK cells. Notably, we found over $95 \%$ of NK cells from both sham and HC lungs were positive for NKG2D (Figure 2I). However, there was an increased density by MFI of NKG2D on NK cells of lungs subjected to HC (Figure 2J, $P=0.005$ ). Following IRI, NKG2D was not increased on other T cell subsets (Supplemental
Figure 1; supplemental material available online with this article; https://doi.org/10.1172/JCI137047DS1). Independent of cytotoxicity, we speculated that IRI might be associated with increased NK cell intracellular IFN- $\gamma$ and TNF- $\alpha$ secretion. However, we found no differences in either IFN- $\gamma$ or TNF- $\alpha$ intracellular cytokine staining in unstimulated NK cells following IRI (Supplemental Figure 2).

Mice lacking NK cells have less acute lung injury. To test if the absence of lymphocytes resulted in reduced lung injury, we performed $\mathrm{HC}$ surgeries on immunodeficient $\mathrm{Il} 2 \mathrm{rg}^{-/} \mathrm{Rag}^{-/} \mathrm{C} 57 \mathrm{BL} / 6$ mice, which lack NK, T, and B cells. Measures of acute lung injury were compared against WT C57BL/6 mice undergoing HC or sham procedures (Figure 3A). Representative $\mathrm{H} \& \mathrm{E}$ histopathology preparations are displayed in Figure 3B and show diffuse alveolar damage (DAD) characterized by hyaline membrane formation, intraalveolar edema, capillary congestion, and neutrophilic infiltration in WT lungs. In contrast, no acute histopathology was observed in sham lungs, and substantially attenuated injury was documented in $\mathrm{Il}_{2 \mathrm{rg}}{ }^{-} \mathrm{Rag}^{-/}$lungs subjected to HC. Quantitative injury measurements agree with these findings. Compared with WT HC lungs, there was reduced lung edema by extravascular lung water volume (EVLW) in WT sham mice and immunodeficient mice (Figure 3C). Additionally, we measured lung vascular permeability after intravenous injection of ${ }^{125}$ I-radiolabeled albu$\mathrm{min}$, and there were less gamma counts recorded per gram of dry 
A

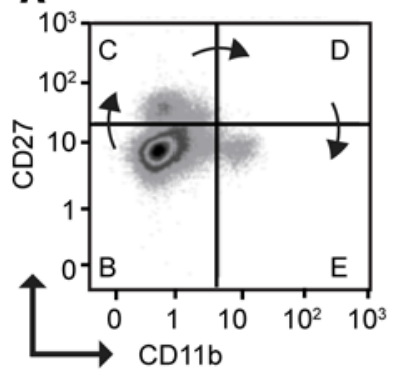

B

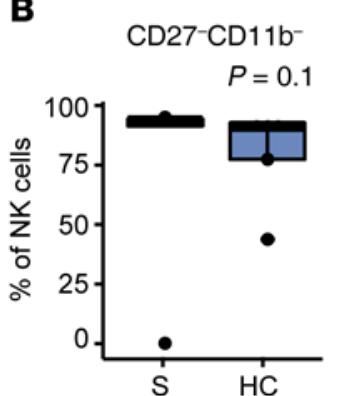

C

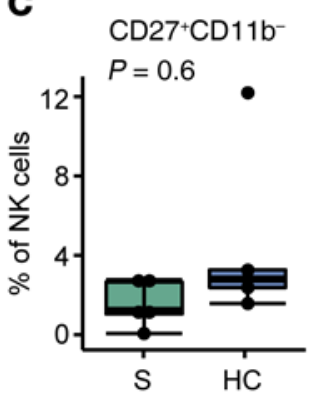

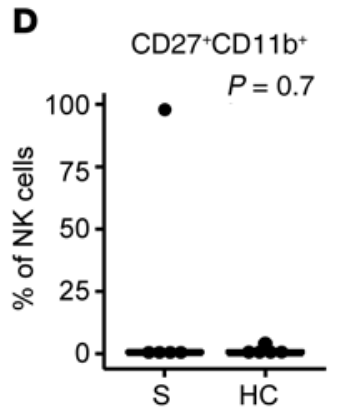

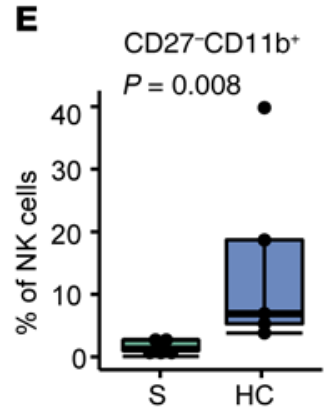

Increasing NK cell maturation
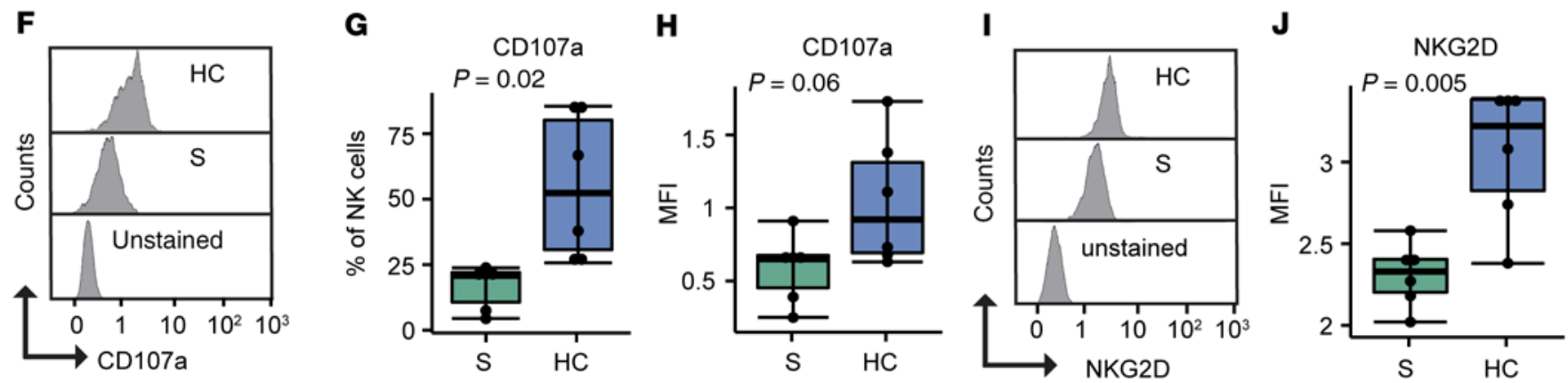

Figure 2. NK cells in acutely injured lungs are more mature, cytotoxic, and activated. (A) As they mature, NK cells (CD3-NKp46+) gain CD27 and then CD11b with the most mature population described as CD27-CD11 b+. There were no differences between $\mathrm{HC}(n=5)$ and sham $(\mathrm{S}, n=5)$ immature NK cell maturation states

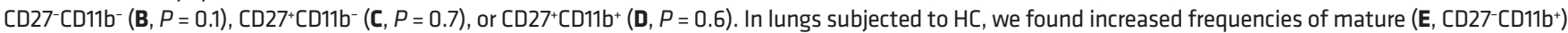
NK cells relative to dissociated sham lungs $(P=0.008)(\mathbf{F})$. The cytotoxic degranulation marker lysosomal-associated membrane protein $(L A M P-1$, CD107a) was measured on NK cells, with fluorescence histograms shown, representative of 6 experiments (C). CD107a ${ }^{+}$NK cells were increased following HC $(n=6)$ relative to sham $(n=6)$ procedure $(P=0.002)$, and the mean fluorescence of CD017a was also increased on NK cells $(\mathbf{H}, P=0.07)$. (I) The stress ligand receptor, NKC2D, was present on $99 \%$ of all NK cells, but had increased surface density after $\mathrm{HC}(\mathrm{J}, n=6, P=0.005)$ compared with sham $(n=6)$. Box and whisker plots show individual data points bound by boxes at 25 th and 75 th percentiles and medians depicted with bisecting lines. Differences were determined by the Mann-Whitney $U$ test.

lung weight in sham lungs and immunodeficient lungs (Il2 $\left.\mathrm{rg}^{-1 /}\right)$ Rag2 $2^{--}$) compared with HC procedures in WT mice (Figure 3D) and less endothelial permeability (Figure 3E). These findings demonstrate that mice lacking NK cells have significantly reduced injury compared with WT mice subjected to experimental IRI.

$N K$ cell adoptive transfer into immunodeficient mice restores acute lung injury. As $\mathrm{Il}_{2 \mathrm{rg}}{ }^{-}-\mathrm{Rag}^{-/}$mice lack $\mathrm{T}$ and B cells in addition to NK cells, we sought to determine if the absence of NK cells alone explained the observed reduction in acute lung injury during PGD. We adoptively transferred $1 \times 10^{6}$ WT C57BL/6 NK cells isolated via negative selection into immunodeficient mice 5 days before $\mathrm{HC}$ experiments. NK cell repopulation of the lung and splenic niches were confirmed via flow cytometry (Supplemental Figure 3). Representative $\mathrm{H} \& \mathrm{E}$ histopathology from $\mathrm{HC}$ lungs following NK cell reconstitution demonstrated severe diffuse alveolar damage (Figure 3B). We found adoptive transfer of NK cells resulted in increased injury relative to WT shams or immunodeficient mice undergoing HC (Figure 3, C and D). As suspected, there was no difference in extravascular fluid volume or gamma counts per gram of dry lung between WT mice and adoptive transfer mice following HC.

NK cells migrate to lung tissue and become activated during experimental IRI. We examined if responding NK cells were intravascular or within the lung tissue at the time of pulmonary IRI using the HC versus sham experimental model. Four hours after clamp release, PE-conjugated anti-NK1.1 was administered intravenously to mice to pulse label the intravascular NK cells (Figure 4A). Dissociated lung cells were then stained for NKp46 expression to identify total NK cells in the tissue. Intravascular and tissue distributions of NK cells among experimental pulmonary IRI and control mice are shown in Figure 4, B and C. In mice undergoing sham procedure, NK cells were mostly anti-NK1.1 and anti-NKp46 costained, indicating that they were in the intravascular compartment (59\%, IQR 57\%-68\%). Following HC procedure, more NK cells had NKp46 staining alone and were within tissue (64\%, IQR 54\%-69\%) compared with the intravascular compartment.

We then compared NK cell maturation states across 2 compartments in WT C57BL/6 mice. Compared with sham, $\mathrm{HC}$ animals had a decrease in the proportion of immature NK cells intravascularly, but the immature fraction in the tissue was unchanged (Figure 4, D and E, with representative flow cytometry dot plots in Supplemental Figure 4). By contrast, the proportion of mature NK cells in the intravascular space was unchanged following $\mathrm{HC}$, but there was a significant increase in mature NK cells in the tissue in $\mathrm{HC}$ versus sham animals (Figure 4, F and G). To visualize NK cells within the lung, we performed tissue immunohistochemistry from $\mathrm{HC}$ and sham lungs (Figure 4H). We found NK cells accumulating bronchioles following IRI. Further, within lungs subjected to HC, it appeared that increased extravasated NK cells may even occlude the small bronchioles. 
A

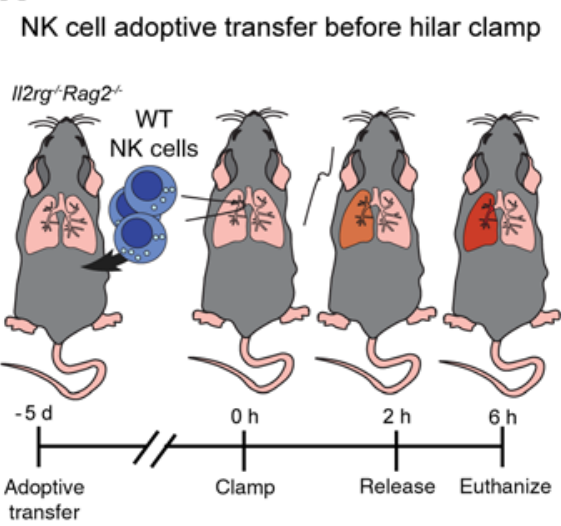

C

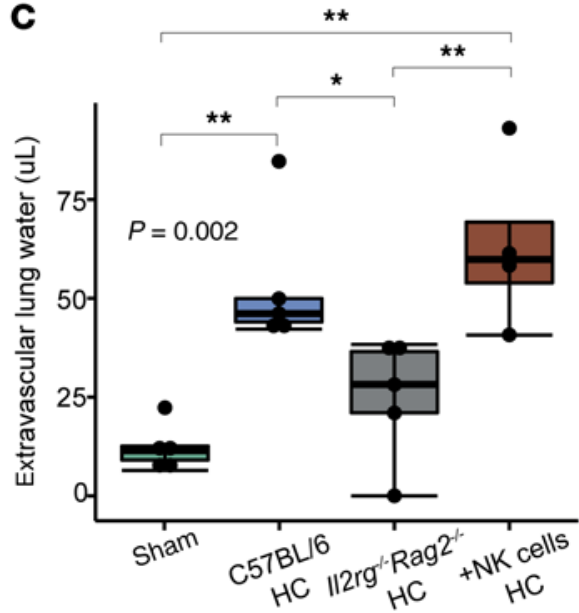

B

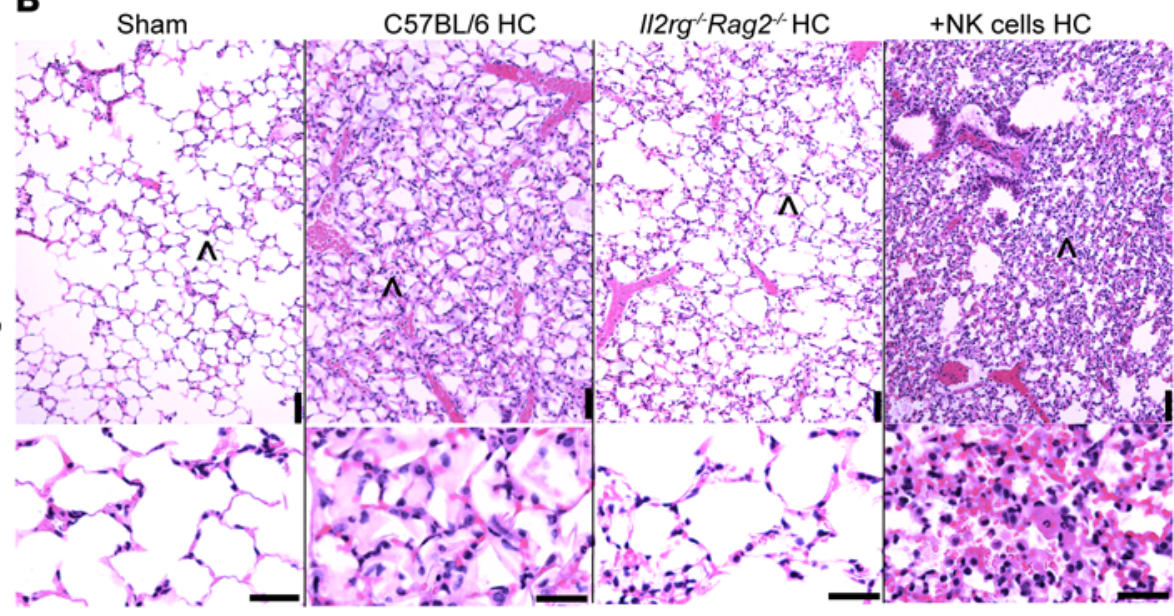

D

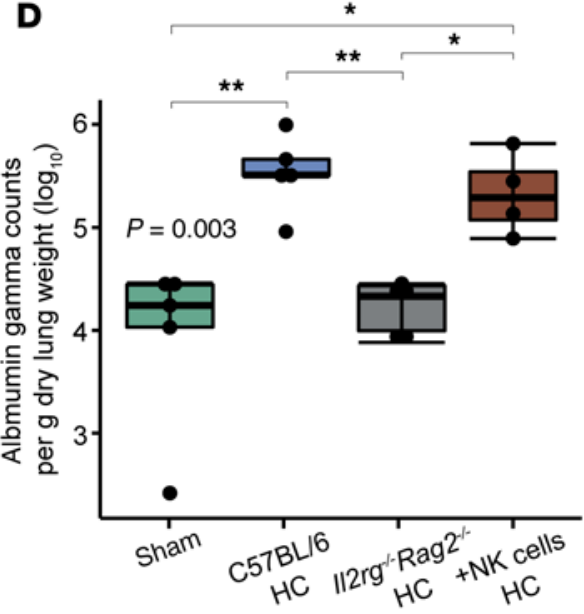

$\mathbf{E}$

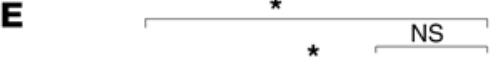

Figure 3. NK cells are sufficient for acute lung injury. (A) $I / 2 \mathrm{rg}^{-1-}$ Rag $2^{-1-}$ mice are deficient in NK, $\mathrm{T}$, and $\mathrm{B}$ cells. Five days preceding procedures, NK cells from WT C57BL/6 mice were adoptively transferred into $/ 12 \mathrm{rg}^{-1-} \mathrm{Rag}^{-1-}$ C57BL/6 mice. (B) H\&E preparations representative of 4 experiments: WT sham

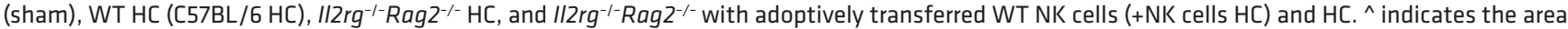
selected for higher magnification (below). DAD, characterized by hyaline membrane formation, intraalveolar edema, capillary congestion, and neutrophilic infiltration, was observed in WT lungs and II $2 \mathrm{rg}^{-1-} \mathrm{Rag}^{-1-}$ lungs reconstituted with WT NK cells. No acute histopathology was observed in sham lungs, and significantly attenuated injury was documented in I/2 $\mathrm{rg}^{-1-} \mathrm{Rag}^{-1-}$ lungs subjected to HC. Scale bars: $100 \mu \mathrm{m}$. Injury metrics are shown in panels C and D with box and whisker plots illustrating individual data points, bound by boxes at 25 th and 75 th percentiles, and with medians depicted with bisecting lines

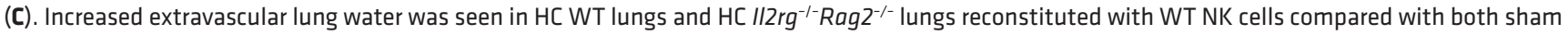
lungs and $\mathrm{HC} 112 \mathrm{rg}^{-1-} \mathrm{Rag2}^{-1-}$ lungs (D). Increased injury by gamma counts in dried lungs following systemic radiolabeled I ${ }^{125}$-albumin treatment were seen in

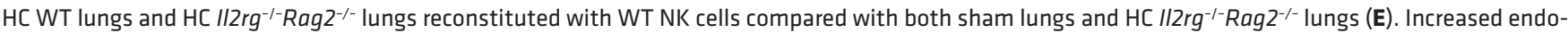
thelial permeability was observed in HC WT lungs and $\mathrm{HC} 1 / 2 \mathrm{rg}^{-1-}$ Rag $2^{-1-}$ lungs reconstituted with WT NK cells compared with sham lungs. All experiments studied 4-5 mice per condition. Differences were assessed by Kruskal Wallis test ( $P$ values shown) and post hoc comparisons with Dunn's test (brackets, $\left.{ }^{*} P<0.05,{ }^{* *} P<0.01,{ }^{* *} P<0.001\right)$. Data are reported from at least 3 independent experiments.

Together, these data demonstrate a tissue-specific increase in mature NK cells following $\mathrm{HC}$ and suggest that NK cells are homing from the periphery to the extravascular space surrounding airways.

Pulmonary NK cells exhibit an activated circulating phenotype. To determine if NK cells in the lung expressed tissue-resident or circulating surface markers, we compared NK cells collected from the dissociated lung with those collected from the blood in $2 \mathrm{HC}$ and 2 sham mice with a 25-parameter spectral flow cytometry panel (Figure 5A). Representative histograms and uniform manifold approximation and projection (UMAP) heat maps for each marker are displayed in Supplemental Figure 5. Notably, we found no difference in tissue-resident markers CD69 or CD49a between blood or lung NK cells (Figure 5,
B and C). However, lung NK cells had decreased CD62L compared with blood NK cells (Figure 5D). This evidence suggests that the lung NK cells originated from circulating sources. Compared with blood NK cells, lung NK cells had increased CD16 (Figure 5E), KLRG1 (Figure 5F), Ly6c (Figure 5G), and CD11b (Figure 5H) and decreased CD27 (Figure 5I) and DNAM1 (Figure 5J) Additionally, TRAIL (Figure 5K), NKG2ACE (Figure 5L), and CD49b (Figure 5M) did not differ between lung and blood. Together, these differences suggest a more activated phenotype. NK cells KLRG1 $(r=0.97, P=0.03)$ and DNAM1 $(r=0.96, P=0.04)$ were positively correlated between lung and blood samples. However, no other markers demonstrated a significant correlation between these compartments. 
A
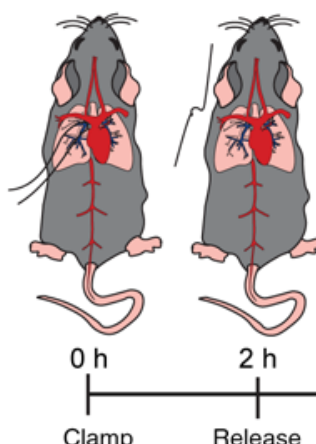

B Total lung NK cells (CD3-NKp46+)

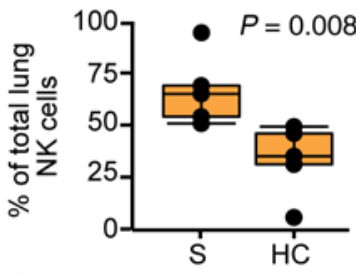

C Tissue (PE-NK1.1 $\left.{ }^{-}\right)$

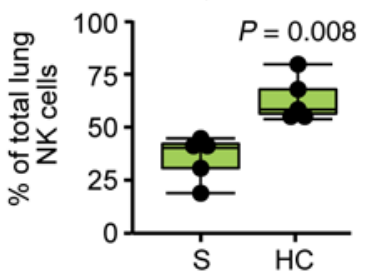

H
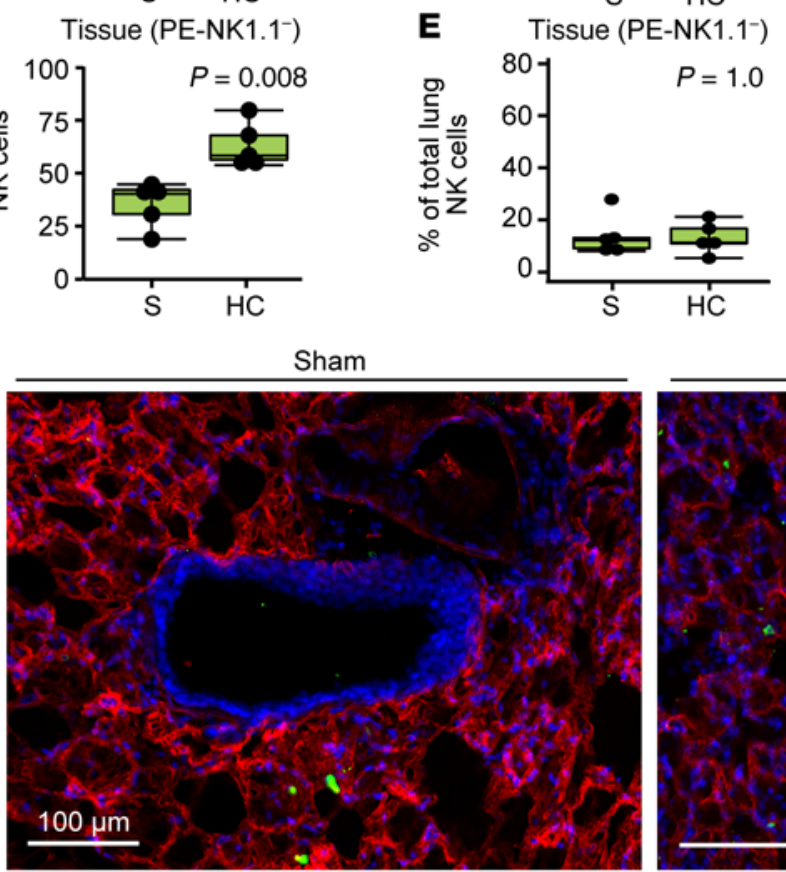
(CD27 ${ }^{-} \mathrm{CD}_{\left.11 b^{-}\right)}$

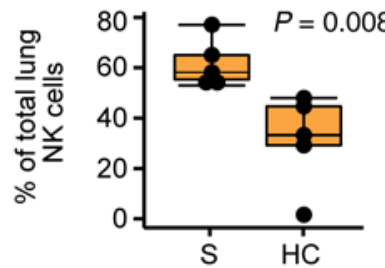

Lung compartment

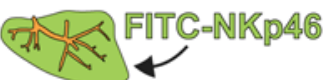

Intravascular (PE-NK1.1 FITC-NKp46+)

Tissue (PE-NK1.1FITC-NKp46+)

5 min later
Intravascular (PE-NK1.1

D Immature lung NK cells $\quad$ F Intravascular (PE-NK1.1+)
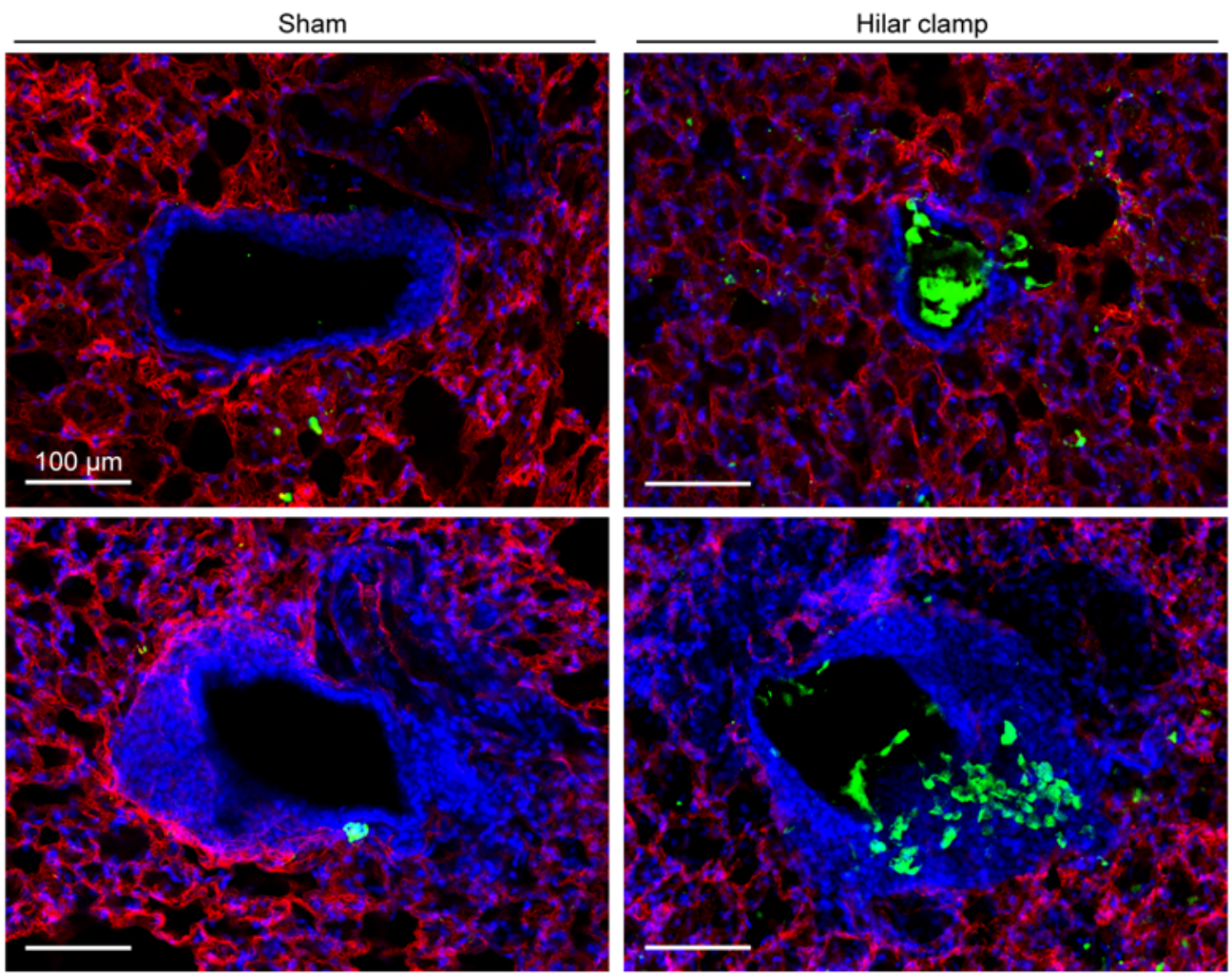
Figure 4. Mature NK cells are found in the lung tissue following experimental IRI. (A) To determine if NK cells were intravascular or in the tissue, PE-conjugated anti-NK1.1 mAb was injected i.v. following HC or sham procedures. In HC lungs, intravascular NK cells were decreased relative to sham lungs (B), and NK cells in the tissue increased (C). (D) Intravascular NK cells were mostly immature, but this population was decreased during HC. (E) In contrast, few tissue NK cells were immature. (F) Similarly, intravascular NK cells had a mature phenotype. (C) Following HC, the NK cells in the lung tissue were more mature than those in the sham lungs. Box and whisker plots show individual data points bound by boxes at 25th and 75th percentiles and medians depicted with bisecting lines. These experiments studied 4-5 mice per condition and differences were assessed with Mann-Whitney $U$ test. (H) To visualize NK cell localization within lung tissue, we performed immunofluorescence. NK cells (NKp46, green) are seen in the extravascular space of bronchioles (nuclei highlighted by DAPI, blue) rather than within the vasculature (CD31, red) of mice undergoing $\mathrm{HC}$ compared with sham lungs.

CD56 $6^{+}$cells are increased in human lung tissue following severe $P G D$. To determine if NK cells are present within lung tissue during clinical PGD, we identified 7 subjects with severe PGD and 6 subjects without severe PGD for whom biopsy tissue blocks were available for research. Surgical lung biopsies from representative subjects with severe PGD are shown at 3,5,20, and 30 days in Figure 6. Biopsies during this critical time after lung transplantation are not routinely performed, but comparison biopsies were obtained from subjects within the first 30 days after transplantation. Baseline characteristics for these subjects are shown in Supplemental Table 1. Figure 6A shows H\&E sections with corresponding high power CD56 staining of biopsy tissue. H\&E preparations demonstrate PGD injury in all 4 samples, with diffuse alveolar damage characterized by edema, diffuse alveolar septal thickening, and type II pneumocyte hyperplasia. CD56 staining reveals abundant CD56 ${ }^{+}$lymphocytes throughout areas of lung injury. $\mathrm{CD}^{+}$and $\mathrm{CD}^{2} 6^{+}$cells were quantified per high power field (HPF, Figure 6, B and C) showing a significantly increased proportion of $\mathrm{CD}^{2} 6^{+}$cells relative to $\mathrm{CD}^{+}$cells during severe PGD (Figure 6D, $P=0.05$ ).

Severe PGD in humans is associated with increased mature $\mathrm{CD}^{+} 6^{+}$ NK cells in bronchoalveolar lavage. To assess if NK cells were associated with clinical PGD, we prospectively collected bronchoalveolar lavage (BAL) fluid from lung transplant recipients within the first 90 days after transplantation during surveillance bronchoscopy. NK cells $\left(\mathrm{CD}^{-} \mathrm{CD} 56^{+}\right)$were assessed by flow cytometry in fresh BAL from 94 bronchoscopies in 61 individual lung transplant recipients. The characteristics of this group of subjects are shown in Table 1 . The frequency of $\mathrm{CD} 16^{+} \mathrm{NK}$ cells were increased during severe PGD, defined as greater than or equal to grade 2 on postoperative day 2 or 3, compared with other grades of PGD (Figure $6 \mathrm{E}, P=0.02)$. Additionally, both the frequency of $\mathrm{CD} 16^{+} \mathrm{NK}$ cells (Figure 6F) and absolute numbers of $\mathrm{CD}^{+} 6^{+} \mathrm{NK}$ cells (Figure 6G) were increased with increasing PGD grade on postoperative day 3 after lung transplantation. Representative histograms for markers on CD16 ${ }^{-}$and $\mathrm{CD}^{+} 6^{+} \mathrm{NK}$ cells are displayed in Supplemental Figure 6A. Compared with CD16- ${ }^{-} \mathrm{NK}$ cells, $\mathrm{CD}^{+} 6^{+} \mathrm{NK}$ cells had less cellular proliferation by Ki67 staining and were more mature, as demonstrated by increased killer cell immunoglobulin-like receptor expression and decreased inhibitory NKG2A receptor expression (Supplemental Figure 6B). These data demonstrate that mature NK cells are increased in transplanted lungs for up to 90 days following PGD, similar to the observations reported in experimental models.

NK cells are also increased in an orthotopic lung transplant with prolonged cold ischemia model of IRI. We measured NK cells in orthotopically transplanted lungs following prolonged cold ischemia (OLT-PCI). Compared with the HC model, this model may more closely replicate the conditions of human organ preservation and transplantation (Figure 7A). Consistent with our HC versus sham experiments, we found increased frequencies of NK cells $\left(\mathrm{CD}^{-}{ }^{-} \mathrm{Kp} 6^{+}\right)$in lungs subjected to OLT-PCI (Figure 7B) as well as increased total NK cells (Figure 7C) relative to native lungs. We also measured $\mathrm{CD}^{+} \mathrm{T}$ cells, NKp46 ${ }^{+} \mathrm{T}$ cells, and myeloid cells in this model. Similarly, we found no differences in frequencies or total counts of these cells in this model (Figure 7, D-I).

The majority of $\mathrm{NK}$ cells are recipient derived and peripheral rather than donor derived or lung resident in the OLT-PCI model. To guide the timing and route of potential lung transplant induction strategies targeting NK cells, we determined whether lymphocytes in the injured lung originate from peripheral populations (recipient) or expand from the pulmonary niche (donor). Following 18 hours of cold ischemia, we transplanted lungs from C57BL/6 Cd45.1 congenic mice into WT (Cd45.2) C57BL/6 mice using the OLTPCI model (Figure 7J). NK cell and other lymphocyte origins were identified by CD 45 allotype (Figure 7K). For reference, splenic cells were $100 \%$ recipient in origin (Figure $7 \mathrm{~L}, P=0.008$ ). We found that more than $80 \%$ of the lymphocytes in the injured lung were of recipient rather than donor origin (Figure $7 \mathrm{M}$ ). Similarly, $70 \%$ of the NK cells in experimental PGD lungs were from the recipient (Figure 7, N and O, IQR 56\%-77\%). Of the donor lymphocytes that were present in the injured lung, 38\% were NK cells (Figure 7O, $P=$ 0.03 ) as compared with $9 \%$ of recipient lymphocytes. Thus, whereas donor NK cells are enriched in the allograft, the majority of NK cells in the allograft following OLT-PCI are recipient derived.

Acute lung injury is abrogated with NK cell depletion preceding experimental IRI. As a preclinical mouse model of an NK cell cytoreduction strategy, NK cells were depleted with anti-NK1.1 monoclonal antibody in both donor and recipient C57BL/6 mice preceding OLT-PCI (Figure 8A). This strategy successfully depleted pulmonary NK cells in the anti-NK1.1 group compared with isotype-matched control Ig-treated lungs (Figure 8B). Mice treated with depleting antibodies had significantly less injury measured by arterial oxygen (Figure 8C), total left lung BAL protein (Figure 8D), and left lung BAL neutrophils (Figure 8E) following OLT-PCI compared with controls. Figure $8 \mathrm{~F}$ demonstrates attenuated injury in representative H\&E images from a mouse subjected to OLT-PCI after receiving anti-NK1.1 antibody relative to an animal that received isotype control. This demonstrates that treatment strategies targeting NK cells may reduce PGD-associated acute lung injury.

Stress molecules are increased on pulmonary epithelial and endothelial cells during experimental IRI. At homeostasis, endothelial and epithelial cells express minimal stress molecules. Under the pressure of hypoxia, HIF-1 $\alpha$ induction leads to rapidly increased endothelial and epithelial stress molecule surface expression (12). This phenomenon has not been investigated during pulmonary IRI, but we hypothesized that the mouse stress-induced NKG2D ligands MULT1 and RAE-1 would be increased within the lung following HC injury. We measured stress ligands on epithelial and endothelial populations 
A

A

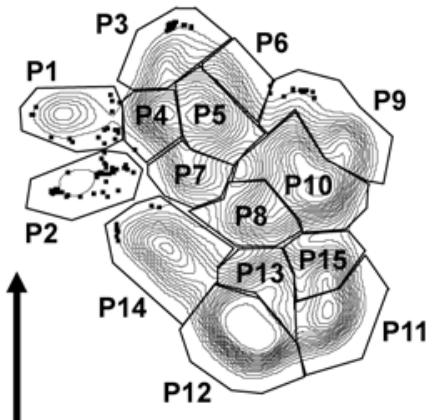

Lung vs. blood
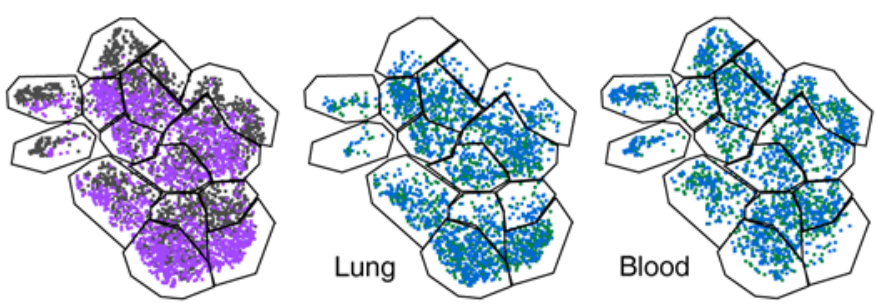

UMAP clusters

P1. CD90.2+CD200R+CD69+TRAIL+CD127+

P2. CD90.2-CD200R+CD69+TRAIL+CD127 ${ }^{+}$

P3. CD90.2+DNAM1+CD627+

UMAP1

$\square$ Lung

Blood

$\checkmark$ Sham

Hilar clamp
P9. CD90.2-CD11 b+KLRG1-NKG2ACE+-

P10. CD62L+CD49b+CD16

P11. CD90.2-KLRG1-Ly6c

P12. KLRG1+Ly6c ${ }^{+} C D 11 \mathrm{~b}^{+}$

P13. KLRG1+Ly6c'NKG2ACE ${ }^{+}$

P14. Ly6c ${ }^{+N K G 2 A C E+C D 90.2+~}$

P15. Ly6c'KLRG1-CD62L+
P4. CD90.2+CD16+NKG2ACE ${ }^{+}$

P5. CD90.2+-CD16+CD627+-

P6. CD16+CD11b-CD627+

P7. KLRG1+CD11b+CD90.2+

P8. KLRG1+CD11b+CD90.2-
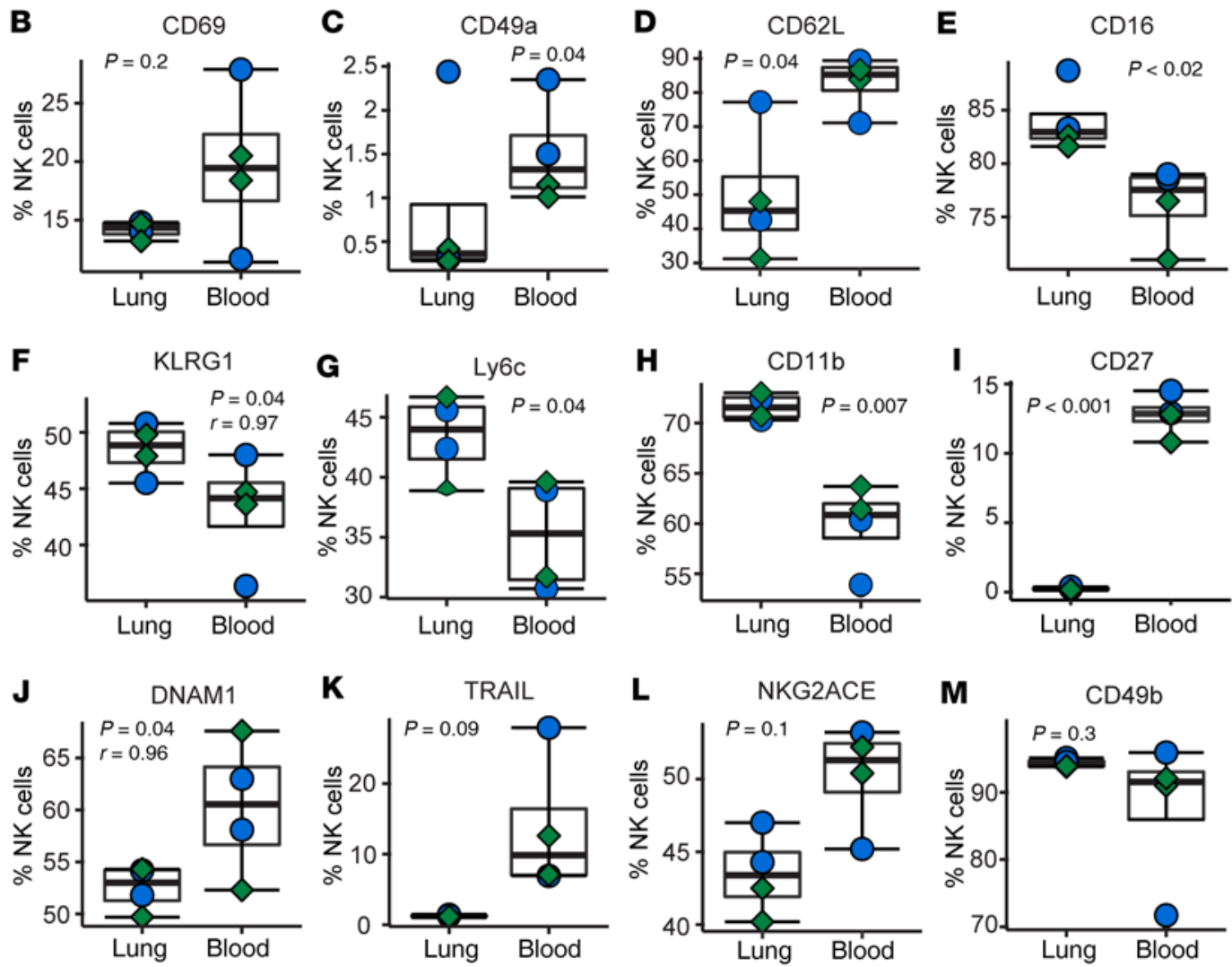

Figure 5. Lung NK cells exhibit a mature phenotype distinct from peripheral blood NK cells. To determine if NK cells expressed tissue-resident markers, lungs and peripheral blood were collected from C57BL/6 mice $(n=4)$ following $\mathrm{HC}(n=2)$ or sham $(n=2)$ procedures. Lung and blood NK cells were phenotypes by spectral flow cytometry and populations were identified based on expression of key markers. (A and B) Cell populations appeared to cluster by tissue origin, but not treatment condition. (C) L-selectin (CD62L) was decreased on lung NK cells relative to blood. Pulmonary NK cells displayed a more activated phenotype with increased CD16 (D), KLRG1 (E), Ly6c (F), and CD11b (C) and decreased CD27 (H) and DNAM1 (I). There were trends for decreased TRAIL (J) and NKC2A/C/E (K) in lung relative to blood NK cells. There was a positive correlation between KLRG1 and DNAM1 in the lung compared with the blood. Box and whisker plots show individual data points bound by boxes at 25 th and 75 th percentiles and medians depicted with bisecting lines. Differences were assessed with paired Student's $t$ tests and statistically significant correlations $(P<0.05)$ between lung and blood NK cells are shown as Pearson's coefficient $(r)$.

within the dissociated mouse lung (Figure 9, A-E). We observed an increased percentage of epithelial cells expressing MULT1 (Figure 9F, $P=0.03$ ), and increased MULT1 median fluorescence intensity on epithelial cells (Figure 9G, $P=0.0002$ ) following HC injury. Sim- ilarly, RAE-1 was increased on epithelial cells as a percentage (Figure $9 \mathrm{H}, P=0.001$ ) and by MFI (Figure 9I). For endothelial cells, MULT1 expression was low and there were no statistically significant differences in the percentage of endothelial cells expressing the molecule 
A Subject \#1 D3

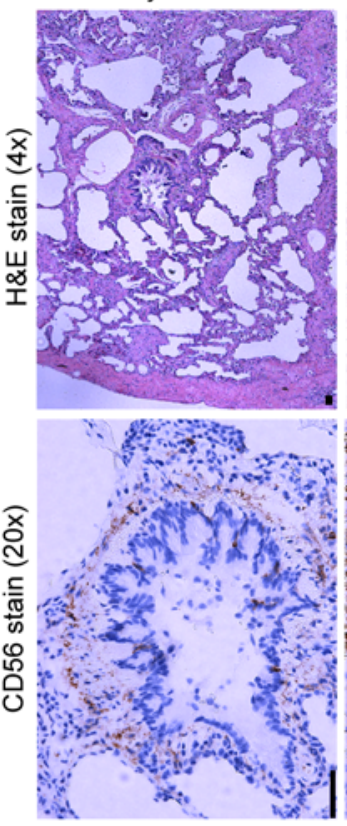

E

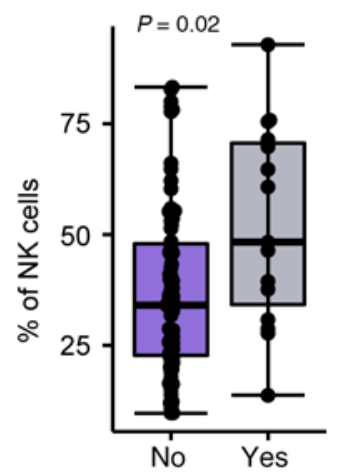

Severe grade $3 P G D$ on day 2 or 3
Subject \#2 D5
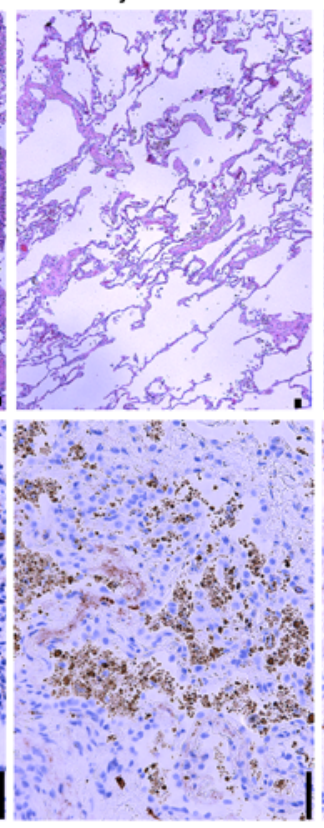

Subject \#3 - D20
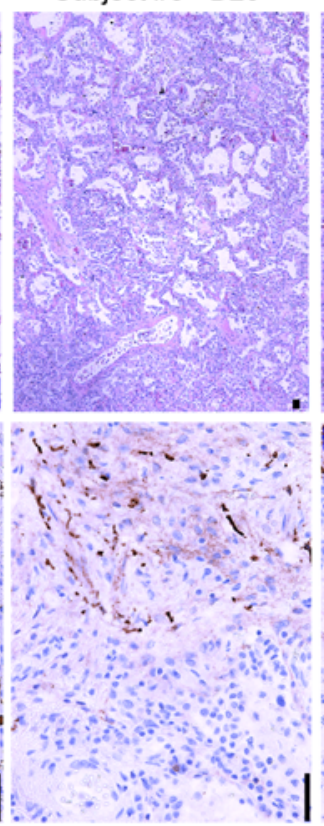

CD16 ${ }^{+}$NK cells

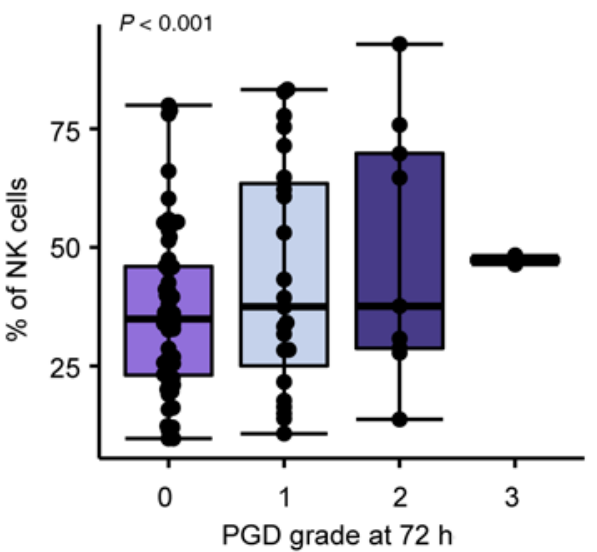

Subject \#4 - D30

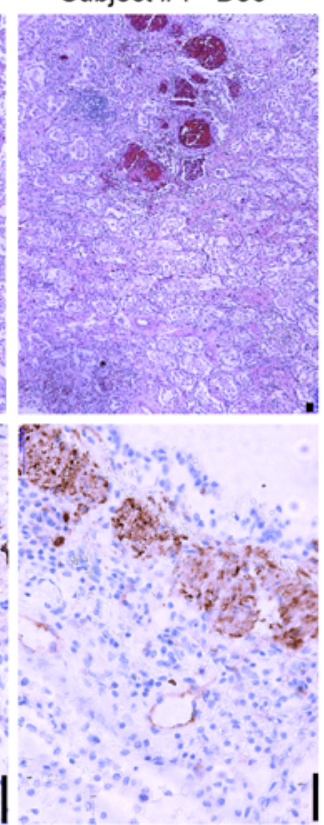

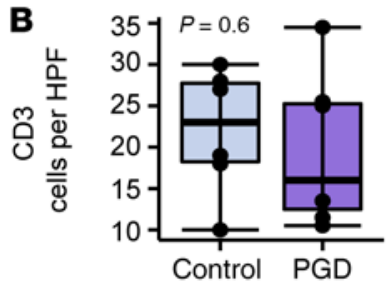

C

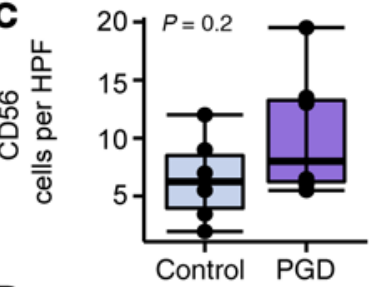

D

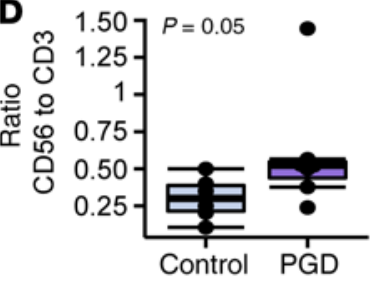

G

Absolute $\mathrm{CD}^{+} 6^{+} \mathrm{NK}$ cells

Figure 6. NK cells are increased and more mature in human allografts following PGD. (A) H\&E and CD56 stains from representative surgical lung biopsies from 4 separate subjects at 3 (D3), 5 (D5), 20 (D20), and 30 (D30) days after lung transplantation. H\&E preparations demonstrate PCD injury of diffuse alveolar septal thickening, edema, and type II pneumocyte hyperplasia. CD56+ cells are observed at sites of injury in each sample. Scale bars: $100 \mu \mathrm{m}$. (B)

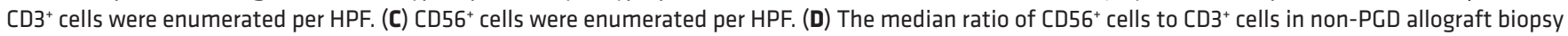
controls $(n=6)$ was 0.3 (IQR 0.22-0.39) and the median ratio of $\mathrm{CD56}^{+}$cells to CD3+ cells in severe PGD $(n=7, P=0.05)$ was 0.52 (IQR $\left.0.44-0.55\right)$. Differences between tissue immunohistochemistry cell counts were assessed with Student's $t$ test. (E) For the first 3 months following lung transplantation, bronchoalveolar lavage was prospectively collected during clinically indicated bronchoscopies. As a percentage of total lymphocytes, CD ${ }^{-}$CD56 ${ }^{+}$CD16 $6^{+}$NK cells were increased with severe PGD at postoperative day 2 or 3 , considered as a binary outcome in generalized estimating equations-adjusted regression ( $P=$ 0.02). A similar increase in absolute numbers of CD16+ NK cells was seen with ordinal severity score for PGD (F, $n=94, P<0.001$ by CLM model). (G) Ordinal PCD severity score also increased as a function of absolute numbers of CD16 ${ }^{+}$NK cells $(P<0.001$ by CLM model). Box and whisker plots show individual data points bound by boxes at 25 th and 75 th percentiles and medians depicted with bisecting lines.

or MFI of MULT1 (Figure 9, J and $\mathrm{K}, P=0.08$ and $P=0.2$ ). RAE- 1 was increased during acute injury by both metrics on endothelial cells (Figure 9L, percentage of cells, $P=0.004$; Figure 9M, MFI, $P=0.03$ ).

Acute lung injury is abrogated in the absence of NKG2D signaling during experimental IRI. Upon binding to stress molecules via the NKG2D receptor, DAP10 and DAP12 signaling led to potent NK cell activation. Given our observations of increased NK cell NKG2D and increased pulmonary parenchymal cell stress ligands, we hypothe- sized that NKG2D ligation is the major activating signal for NK cells following IRI. Figure 9N depicts the 4 experimental groups used to investigate the NKG2D receptor as an activating signal in the $\mathrm{HC}$ versus sham model. To determine if NKG2D ligation was the integral activating signal, we dosed animals with a nondepleting antibody against the NKG2D receptor or an isotype-matched control antibody on days -7 and -1 preceding $\mathrm{HC}$ procedures. To determine if the NKG2D receptor on NK cells, versus other NKG2D-expressing 


\begin{tabular}{ll}
\hline \multicolumn{2}{l}{ Table 1. Characteristics of the $\mathbf{6 1}$ human subjects } \\
Variable & $\boldsymbol{n}$ \\
Sex, male/female & $39 / 22$ \\
Double lung transplant & 54 \\
Ethnicity & \\
White & 40 \\
African American & 5 \\
Asian and Pacific Islander & 11 \\
Other & 5 \\
Transplant group & \\
$\quad$ COPD & 7 \\
Cystic fibrosis & 7 \\
Interstitial lung disease & 47 \\
PCD grade at 72 hours & \\
0 & 38 \\
1 & 10 \\
2 & 8 \\
3 & 5 \\
\hline
\end{tabular}

cells, was mediating this interaction, we adoptively transferred NK cells from mice lacking the NKG2D receptor $\left(\mathrm{Klrk1}^{-1-}\right)$ or from WT C57BL/6 mice into immunodeficient mice (Il2 $\left.\mathrm{rg}^{-/-} \mathrm{Rag}^{-1-}\right) 5$ days preceding HC. In animals with NKG2D antibody blockade or NK cells lacking the NKG2D receptor, we found reduced extravascular lung water (Figure 9O) compared with the 2 control groups. Similarly, there was a reduction in radiolabeled albumin detected in the lungs of mice that received anti-NKG2D or $\mathrm{Klrk1}^{--} \mathrm{NK}$ cells (Figure 9P). Finally, we found significantly decreased endothelial cell permeability in the group receiving anti-NKG2D monoclonal antibody (Figure 9Q). These results demonstrate that the NKG2D receptor contributes to IRI that is driven by NK cells.

\section{Discussion}

Together, these data demonstrate a central role for NK cells in acute lung injury resulting from ischemia, hypoxia, and reperfusion. Under the stress of experimental IRI, we observed upregulation of stress molecules on endothelial and epithelial cells and evidence of activated NK cells trafficking from the periphery to lung tissue in mouse models and human tissue samples. We demonstrated that these NK cells are cytotoxic and sufficient for the lung injury of pulmonary IRI. Our finding that NK cell depletion abrogated acute lung injury due to experimental IRI, suggests that NK cells have potential as therapeutic targets. We further identify a plausible specific target, the NKG2D receptor.

Our data suggest that a dominant mechanism for this interaction is NK cell recognition via NKG2D of epithelial and endothelial stress molecules within the lung. These stress molecules, which are ligands for the NKG2D receptor, have been shown to be increased in the lung or on pulmonary epithelium during idiopathic pulmonary fibrosis, exposure to tobacco smoke, and with acute infection (20-22). While additional mechanisms of NK cell activation, such as through the NKp46 receptor, may contribute to IRI injury (23), our finding that targeted NKG2D genetic depletion and NKG2D blockade reduce experimental PGD injury suggest that NKG2D antibody blockade may be useful as a potential human therapy.
Indeed, an analogous monoclonal anti-NKG2D antibody is currently undergoing phase II clinical trials for the treatment of inflammatory bowel disease (24). We observed an increase in NKG2D on NK cells in our HC 4-hour injury model. This was somewhat surprising, as NKG2D is reported to be endocytosed following ligation via DAP10/12-dependent mechanisms (25) and NKG2D is downregulated on NK cells with constitutive stress ligand signaling (26). However, this initial increase in NKG2D might reflect an influx of activated circulating NK cells. We noted that with longer NKG2D-ligand interaction time, as occurs in the OLT-PCI model, there was relatively less NKG2D observed on NK cells.

NK cells are attractive targets for novel therapeutics in transplantation. NK cell receptors are somatically encoded and not shared on many other significant immune cell subsets. At the same time, they have a potential to profoundly impact transplant-related lung injury, because their IFN transcripts and cytotoxic granules are abundant and preformed, rendering them resistant to induction immunosuppressants $(27,28)$. There also is evidence that they may become even more activated by scavenging IL- 2 in the setting of IL-2 receptor blockade (29).

We found that a substantial proportion of lymphocytes and NK cells in the injured lung are of recipient or peripheral origin. Intravascular pulse labeling experiments and tissue immunohistochemistry demonstrate that most of these NK cells are within the injured lung tissue rather than the intravascular space. Given the rapid time course and predominantly recipient origin, most NK cells appear to be homing to the allograft, rather than proliferating locally. Donor-derived NK cells may originate from transplanted lymphoid tissue. Further, these IRI-associated NK cells have more activated, mature phenotypes and lack tissue-specific markers. Overall, these findings are consistent with NK cell behavior in viral infections and suggest a conserved response by NK cells to lung tissue injury across etiologies (30). We found decreased PE-labeled NK cells in the injured lungs, and we believe this approach accurately assessed the NK cell compartment, as injured tissue may be more permeable to PE-labeled anti-NK1.1. Interestingly, the injury phenotype appeared somewhat accentuated in adoptive transfer experiments. This increased injury may indicate that NK cells are activated during adoptive transfer with unopposed proliferation in the relatively empty pulmonary niche. One outstanding question is how NK cells may traffic to sites of injury. Soluble stress ligands may serve as NK cell chemokines (31). NK cells may also be responding to chemokines associated with PGD, via chemokine receptors CCR2, CCR5, and CXCR3 $(32,33)$.

The major mechanism of injury appeared to be direct cytotoxicity, given evidence of cytotoxic degranulation without changes in intracellular cytokine levels. Nonetheless, we did not examine stimulated cytokine release and this remains a potential mechanism, as NK cells are potent recruiters and activators of other immune cells through chemokine secretion (34). NK cells can also mediate sterile lung injury induced with lipopolysaccharide via neutrophil recruitment (35). Several studies of experimental and human PGD have described the importance of neutrophils, via neutrophil-derived extracellular traps (NETs), as well as macrophages in mediating PGD lung injury $(8,36$, 37). Chemokine release may recruit neutrophils and bridge the role of NK cells with our prior finding of NET-mediated PGD injury $(8,38)$.

In human lung transplant BAL, we found that $\mathrm{CD} 16^{+} \mathrm{NK}$ cells were increased in absolute and relative quantities for up to 90 days 
A

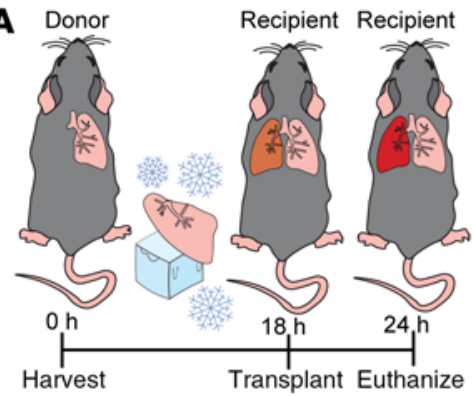

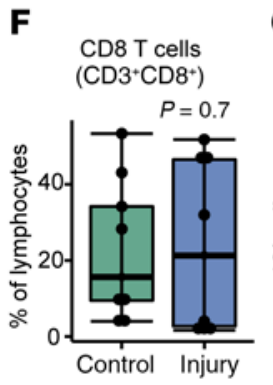

G

CD8 T cells (CD3 ${ }^{+} \mathrm{CD}^{+}$)

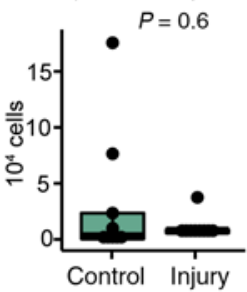

B

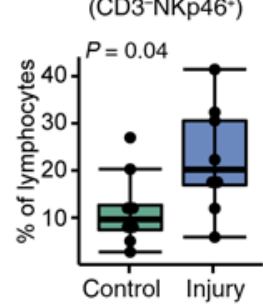

C

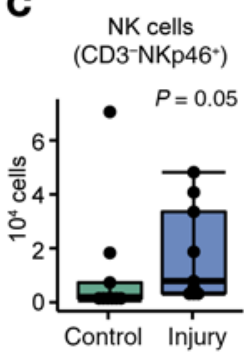

D
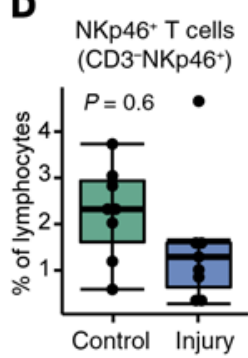

E

NKp46 $6^{+}$cells (CD3*NKp46+)

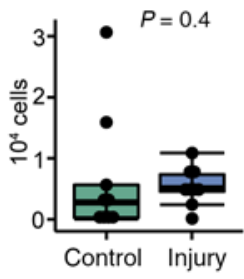

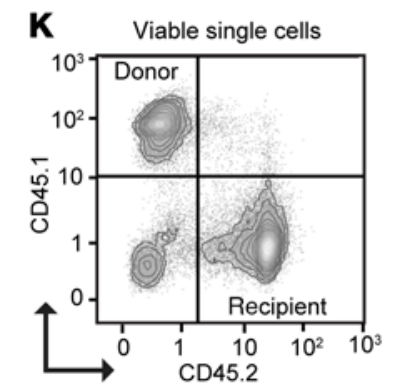

$\mathbf{L}$

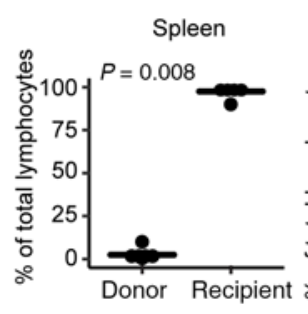

H

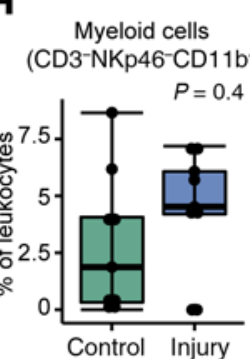

I Myeloid cells
(CD3-NKp46-CD11b*) $P=0.6$
J CD45.1 Donor

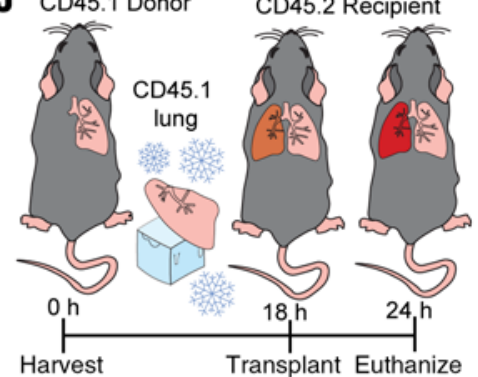

M

N
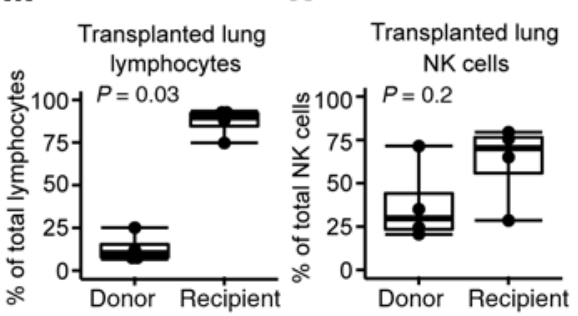

O

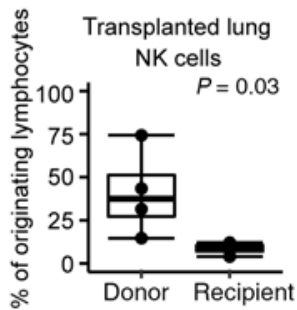

Figure 7. NK cells are increased in a translational OLT-PCI model. (A) In the OLT-PCI model, donor lungs were harvested, stored for 18 hours at $4^{\circ} \mathrm{C}$, and transplanted for 8 hours of in situ warm reperfusion before mice were euthanized. (B) NK cells (CD3-NKp46+) were increased in dissociated lung tissue (HC) relative to sham procedures as a percentage of lymphocytes and (C) by total cell count. There were no differences in NKp46 ${ }^{+} T$ cells ( $\mathbf{D}$ and $\left.\mathbf{E}\right), \mathrm{CD}^{+} \mathbf{T}$ cells (F and $\mathbf{G}$ ), or myeloid cells (H and I). (J) In the same OLT-PCI model, CD45.1 allotype donor lungs were transplanted into CD45.2 allotype recipient mice following prolonged cold ischemia. (K) Donor cells were identified by CD45.1 expression and recipient cells were identified as by CD45.2 surface expression. (L) Splenic lymphocytes were of recipient origin. (M) More than $80 \%$ of transplanted lung lymphocytes were also from the recipient. (N) In the injured lung, $70 \%$ of the NK cells were of recipient origin. (0) The donor NK cells represented $30 \%$ of the donor lymphocytes, which was significantly more than the proportion of recipient NK cells. C57BL/6 donor and recipient mice were given depleting anti-NK1.1 or isotype-matched control monoclonal antibodies at 7 days and 24 hours preceding OLT-PCI. Differences were assessed using Student's $t$ test. Each condition studied at least 5 mice.

following severe PGD. CD16 is an activating Fc receptor that initiates antibody-dependent cell-mediated cytotoxicity (ADCC) (39) as well as a marker of NK cell maturation. Lung-restricted autoantibodies have been shown to mediate experimental PGD and are significant risk factors for clinical PGD (40-42). These are not part of routine pretransplant screening, but in one series of 142 lung transplant recipients, up to $30 \%$ of subjects had at least one lung-restricted autoantibody (36). Together, these data suggest that ADCC from donor-specific alloantibodies or lung-restricted autoantibodies may be another potential mechanism for NK cell injury in PGD.

A significant strength of this study is the concordant findings from 2 separate animal models of PGD paired with associations among a single-center human lung transplant cohort. Nonetheless, this work has some limitations: obtaining tissue samples early in subjects in the posttransplant period is not feasible as these patients can be critically ill and subject to increased risk from invasive sampling. Subjects with PGD often have less early invasive surveillance bronchoscopies for this reason, which would be expected to bias our human BAL findings towards the null hypothesis. Results from a single-center human study are subject to variability from differences in transplant protocols and heterogeneity in subject characteristics. While we show NK cell-specific effects, we cannot exclude the possibility that a small number of innate lymphoid cells may participate (Supplemental Figure 7L) (43). A recent study of donor lung tissue before and after reperfusion showed a steep decline in highly immature NK cells in lungs (CD3-CD11b-CD11 $\left.{ }^{-} \mathrm{CD} 56^{+}\right)$preceding PGD, which indirectly supports our findings here (44).

Our findings may have broader implications, as NK cells can play multiple important roles in the context of lung transplantation (45). In addition to the effects observed on IRI, NK cells appear to impact CLAD outcomes both through immune surveillance of CMV (46) and depletion of host antigen-presenting cells (47). While NK cell depletion could increase infection or rejection risks, targeting NK-associated receptors most associated with IRI, such as NKG2D, 
A

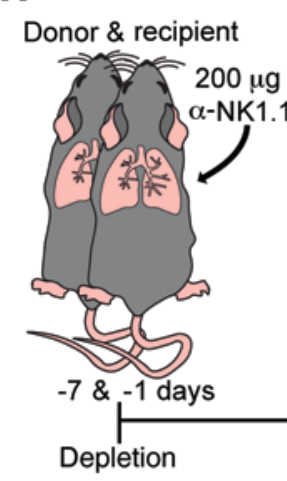

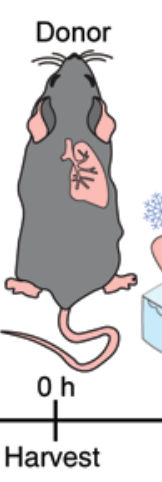

B

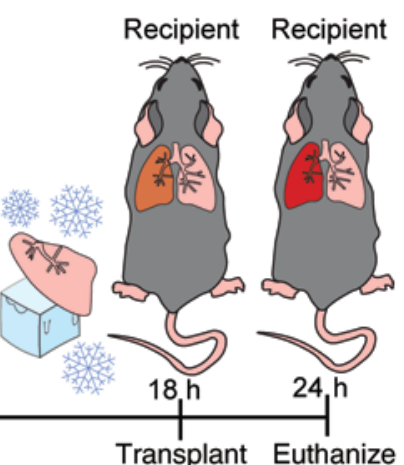

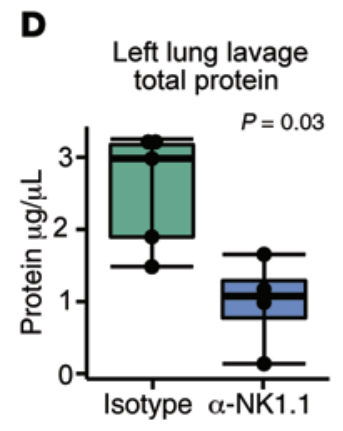

E

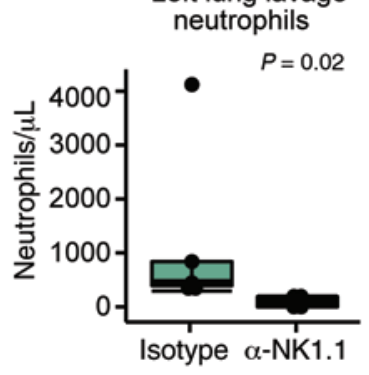

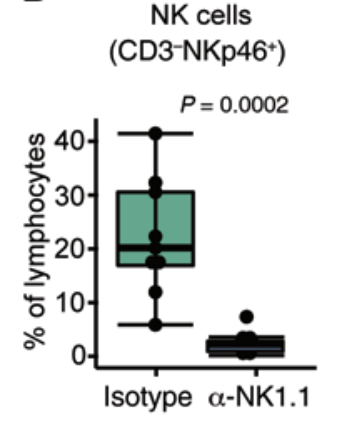

$\mathbf{F}$

C

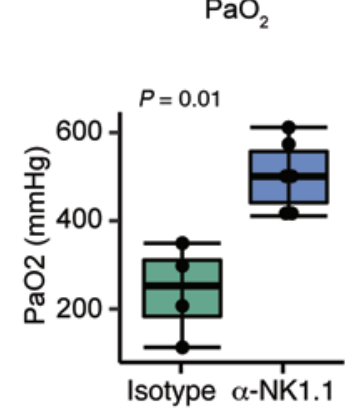

Isotype H\&E

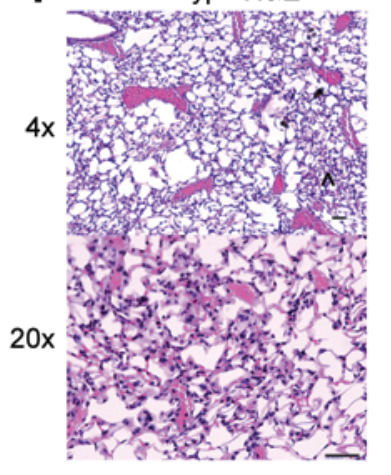

$\alpha-\mathrm{NK} 1.1$

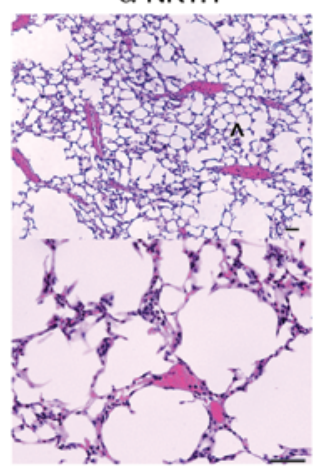

Figure 8. NK cells contribute to injury in a translational OLT-PCI model. (A) C57BL/6 mice were given anti-NK1.1 or isotype-matched control antibody at 7 days and 1 day preceding OLT-PCI. (B) NK cells were successfully depleted from the lungs of animals receiving anti-NK1.1. Lung injury as measured by arterial $\mathrm{PaO}_{2}$ (C), left lung bronchoalveolar lavage protein (D), and left lung bronchoalveolar lavage neutrophil count (E) was significantly decreased in mice with NK cell depletion preceding OLT-PCI compared with isotype control mice. (F) H\&E sections of 4 representative experiments are shown at $\times 4$ and $\times 20$ magnification from isotype and anti-NK1.1 lungs following OLT-PCI. Scale bars: $100 \mu \mathrm{m}$. ^ indicates the area selected for higher magnification (below). Differences were assessed using Student's $t$ test. Each condition studied at least 5 mice.

could ameliorate this concern. One outstanding question is whether potential treatment should be targeted within the donor organ or as part of the recipient induction regimen. Our finding that NK cells are mostly recipient derived would suggest adding an NK cell inhibitory therapy to induction protocols may be beneficial.

In summary, we describe a novel role for NK cells in experimental IRI and lung PGD, which implicates the NKG2D receptor as a potential therapeutic target that could improve outcomes following lung transplantation.

\section{Methods}

Mice. Male mice aged 8 to 12 weeks and weighing $28-30 \mathrm{~g}$ were housed in a pathogen-free barrier facility for all experiments. C57BL/6J and CD45.1 (B6.SJL-Ptprc ${ }^{a} P e p c^{b} /$ BoyJ) congenic animals were purchased from Jackson Laboratories (catalog 002014). Il2 $\mathrm{rg}^{-/} \mathrm{Rag}^{-/-}$ constitutive double knock-out C57BL/6 mice (C57BL/6NTac; B1O (Cg)

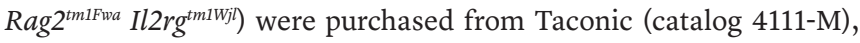
and $\mathrm{C} 57 \mathrm{BL} / 6 \mathrm{Klrk1}^{-/-}$mice were derived as previously described (26). All experiments were conducted according to protocols approved by the UCSF Institutional Animal Care Use Committee.

Experimental models of pulmonary IRI. Details regarding methods of experimental pulmonary IRI models have been previously published $(8,38)$. Mouse procedures were performed by a surgeon with microvascular training. For the HC model, mice underwent left thoracotomy to expose the hilar structures. A silk suture was tied in a slipknot or left untied in a sham (S) surgery. Mice were extubated and monitored until complete recovery from anesthesia. Following a 2-hour period of ischemia, the suture was removed, and animals were euthanized after 4 hours of warm reperfusion. In a series of experiments, $20 \mu \mathrm{g}$ PE-conjugated anti-NK1.1 (clone PK136, BioLegend, catalog 108708) in sterile PBS was injected retro-orbitally in anesthetized mice 10 minutes prior to euthanasia. Lungs were collected and dissociated, and NK cells were analyzed by flow cytometry.

Orthotopic left lung transplantations in mice with OLT-PCI of the donor organ were performed as previously reported $(8,48)$. The left donor lung was air-inflated, infused with Perfadex (XVIVO Perfusion Inc.), and stored at $4^{\circ} \mathrm{C}$ for 18 hours while wrapped in Perfadex-soaked gauze. The donor organ was implanted using a plastic cuff for anastomosis. The recipient animal was euthanized 8 hours after transplantation. The left and right lungs, as well as blood, were collected for analysis.

In selected experiments, B6 Cd45.1 mouse lungs (B6.SJL-Ptprc ${ }^{a} P e p c^{b}$ / BoyJ) were transplanted into C57BL/6 recipients following prolonged cold ischemia. NK cells of CD45.1 or CD45.2 allotype origin were enumerated by flow cytometry in dissociated lung and spleen. Dissociated native and uninjured lungs were used as internal flow cytometry controls.

NK cell depletion and NKG2D blockade. C57BL/6 donor and recipient mice were injected i.p. with $200 \mu \mathrm{g}$ anti-NK1.1 monoclonal antibody (clone PK136, American Type Culture Collection, catalog HB-191) or isotype-matched control antibody (anti-rat, gp42, clone 3G7, provided by Mary Nakamura, University of California, San Francisco, 

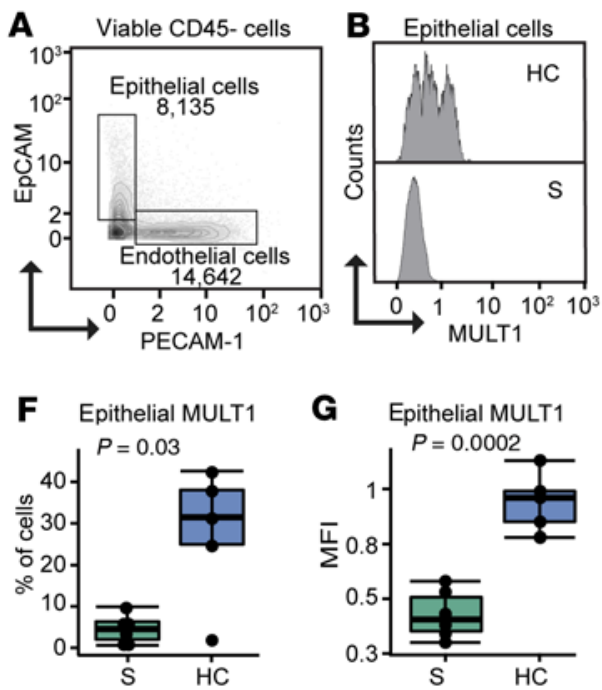
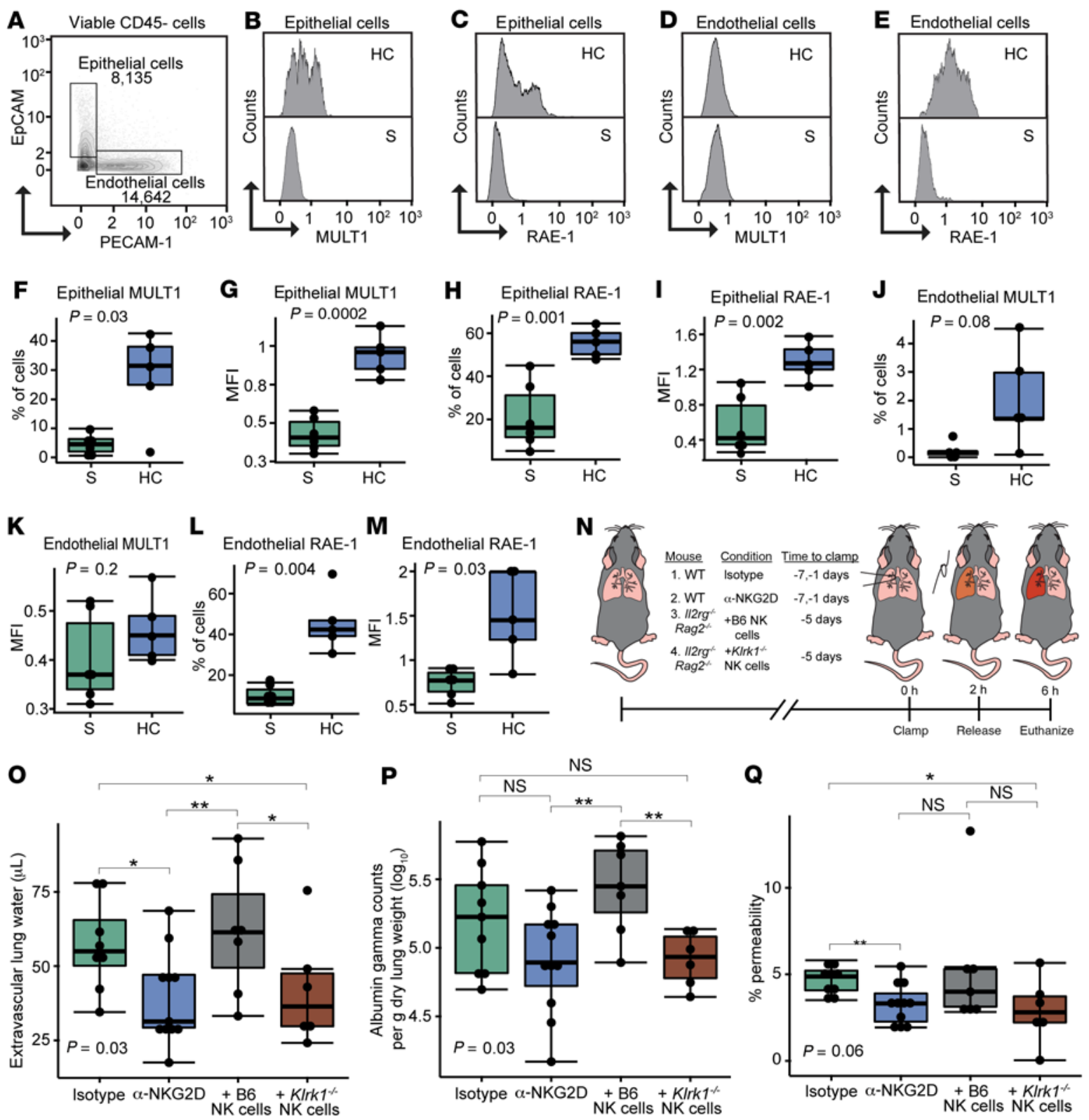

Figure 9. Recognition of stress molecules increased by IRI through the NKC2D receptor contributes to IRI pathology. (A) Following HC and sham procedures, epithelial cells (CD45-EpCAM+) and endothelial cells (CD45-PECAM-1+) were identified in dissociated lung. (B-E) MFI and percentage of cells expressing MULT1 and RAE-1 were measured. MULT1 expression on epithelial cells was increased as a percentage (F) and by MFI (C) following HC. (H, I) The RAE-1 stress ligand was also increased on epithelial cells. (J, K) Endothelial MULT1 was not significantly different between HC and sham procedures. (L, M) Endothelial RAE-1 was increased following HC versus sham procedures. (N) Four conditions demonstrated the role of the NKC2D receptor in the HC model: (a) isotype control antibody or (b) NKC2D-blocking antibody was administered 7 days and 1 day preceding HC; (c) C57BL/6 NK cells or (d) NKC2D knock-out $\left(\mathrm{KIrk}^{-/-}\right) \mathrm{NK}$ cells were adoptively transferred into immunodeficient mice (II2 $\left.\mathrm{rg}^{-/-} \mathrm{Rag}^{-/-}\right)$. Quantitative injury metrics were assessed for each condition and displayed as (0) extravascular lung water, (P) gamma counts of radiolabeled ${ }^{125}$-albumin per gram of dry lung, and (Q) endothelial permeability. Box and whisker plots show individual data points bound by boxes at 25th and 75th percentiles and medians depicted with bisecting lines. Flow cytometry experiments studied 5 mice for each condition, antibody experiments studied 9-10 mice per condition, and adoptive transfer experiments studied 6-7 mice per condition. Comparisons between 2 groups were made with the Mann-Whitney $U$ test, while group differences were assessed with the Kruskal-Wallis test using Dunn's test for post hoc comparisons.

San Francisco, CA) at 7 days and 24 hours preceding surgery (49). For HC experiments, C57BL/6 mice were injected with $200 \mu \mathrm{g}$ antiNKG2D monoclonal antibody (clone CX5, UCSF antibody core, catalog AM053-PURE) or isotype-matched control antibody (rat IgG1, clone HRPN, UCSF antibody core, catalog AM047-PURE) at 7 days and 24 hours preceding $\mathrm{HC}$ procedures.
Acute lung injury measurements. Wet-to-dry lung weights, lung vascularpermeability to ${ }^{125}$ I-labeled albumin (Iso-Tex Diagnostics) delivered i.v., and extravascular lung water were measured as previously described (8). Arterial blood partial pressure of oxygen $\left(\mathrm{PaO}_{2}\right)$ was assessed after mice were first anesthetized i.p. with $0.6 \mathrm{mg} / \mathrm{kg}$ ketamine and $0.1 \mathrm{mg} / \mathrm{mL}$ xylazine. Anterior tracheotomy was performed, and animals were supported 
with $100 \% \mathrm{O}_{2}$ as previously described (8). After 5 minutes, arterial blood was collected via left ventricular puncture and $\mathrm{PaO}_{2}$ was measured with an i-STAT 1 Handheld Analyzer and VetScan CG4 i-STAT Cartridges (Abaxis, catalog 89126). Additionally, single left lung lavage with saline was performed, and total protein and neutrophils were measured (8).

To visualize lung injury, mice were euthanized following IRI procedures. The lungs were inflated with air, and the bronchus was tied off with silk suture. The inflated lung was excised from the chest cavity and placed into $10 \%$ neutral buffered formalin for 48 hours, then transferred to $70 \%$ ethanol. The lungs were embedded in paraffin, sectioned, and stained with H\&E. Slide images were acquired with an Olympus BX51 microscope at $\times 4$ and $\times 20$ magnifications using a digital camera (Diagnostic Instruments, DBX) and commercial imaging software (Bioquant Life Science 2013 v13.5.60).

Lung digestion. To isolate cells from harvested lungs, the left lungs were added to a solution containing $1 \mathrm{~mL}$ PBS with $0.05 \mathrm{mg} / \mathrm{mL}$ Liberase (Sigma-Aldrich, catalog 5401119001) and $10 \mu \mathrm{L}$ of $10 \mu \mathrm{g} /$ $\mu \mathrm{L}$ DNAse I (Sigma-Aldrich, catalog 10104159001). The lungs were cut with scissors into $1 \mathrm{~mm}$ pieces and incubated in a shaking incubator for 1 hour at $37^{\circ} \mathrm{C}$. The incubated lungs were strained through a $40-\mu \mathrm{m}$ mesh nylon filter using $10 \mathrm{~mL}$ PBS and pelleted for 5 minutes at $4^{\circ} \mathrm{C}$. The samples were resuspended in $2 \mathrm{~mL}$ PBS and $2 \mathrm{~mL} \mathrm{ACK}$ Lysis Buffer (Gibco, catalog A10492) for 5 minutes at room temperature, then pelleted for 5 minutes at $4^{\circ} \mathrm{C}$. Each sample was then washed and resuspended in flow cytometry buffer.

Mouse lung immunophenotyping. Following collagenase digestion, suspended cells were washed and incubated at room temperature with anti-mouse anti-CD16/CD32 to block nonspecific binding and stained with viability dye (Fixable Viability Dye eFluor506, eBioscience, cata$\log$ 65-0866-14). Lymphocyte subsets were identified using Alexa Fluor 700-conjugated anti-CD45 (clone 30-F11, BD Biosciences, catalog 560510), APC-conjugated anti-CD3 (clone 17A2, BioLegend, catalog 100235), PE-Cy7-conjugated anti-CD8 (clone 53-6.7, BioLegend, catalog 100721). NK cell subsets were identified using FITC-conjugated anti-CD335 (NKp46) (clone 29A1.4, BioLegend, catalog 137606), PE-conjugated anti-CD314 (NKG2D) (clone CX5, BioLegend, catalog 130207), PerCP-conjugated anti-CD27 (clone LG.7F9, Invitrogen, catalog 46-0271-80), and V450-conjugated anti-CD11b (clone M1/70, BD Biosciences, catalog 560456). For intracellular cytokine measurements in NK cells, lung cells were prepared with permeabilization and fixation buffers per manufacture protocol (BD Biosciences, catalog 554714). Alexa Fluor 647-conjugated anti-IFN- $\gamma$ (clone XMG1.2, BioLegend, catalog 505816) and PE-Dazzle 594-conjugated anti-TNF- $\alpha$ (clone MP6-XT22, BioLegend, catalog 506345) stains were applied (representative histograms in Supplemental Figure 2). The core panel and gating strategy are shown in Supplemental Figure 7. A separate NK cell degranulation panel employed V450-conjugated anti-CD107a (clone 1D4B, BD Biosciences, catalog 560648). Samples were acquired with a Beckman Coulter Navios cytometer (50). Negative thresholds were set based on fluorescence minus one and unstained controls. Antibody staining was quantified by MFI and visualized by logarithmically scaled histogram plots. Viable lymphocytes were identified by e506- ${ }^{-} \mathrm{CD} 45^{+}$ expression in addition to forward and side light scatter profiles. NK cells were identified as CD3-NKp46 ${ }^{+}$. Intravascular NK cells were identified as CD3-NKp46 ${ }^{+} \mathrm{NK} 1.1^{+}$and NK cells in lung tissue, which would not be stained with the injected PE-conjugated anti-NK1.1 mAb, were identified as $\mathrm{CD}^{-} \mathrm{NKp} 46^{+} \mathrm{NK} 1.1^{-}$(51). The antibody conjugates, data acquisition, and gating strategy for the spectral flow cytometry experiments are detailed in the supplemental methods and Supplemental Figure 8. $\mathrm{CD}^{4} 5^{+} \mathrm{CD} 3^{-} \mathrm{NKp} 46^{+} \mathrm{NK}$ cells are highlighted in this extended panel, which was designed to exclude innate lymphoid, T, and B cells.

Tissue immunofluorescence. Fixed lung cryosections were prepared as previously described (52). Off-target binding was blocked with $10 \%$ normal donkey serum with $0.3 \%$ BSA in staining buffer $(0.3 \%$ Triton $\mathrm{X}-100$ in PBS), and samples were incubated overnight with primary antibodies raised against NKp46 (goat polyclonal, R\&D Systems, catalog AF2225) or CD31 (rabbit monoclonal D8V9E, Cell Signaling Technology, catalog 77699S). Samples were washed and then incubated overnight with Alexa Fluor 488-conjugated donkey anti-goat IgG (polyclonal, Jackson ImmunoResearch, catalog 705-545-147) and Cy3-conjugated donkey anti-rabbit IgG (polyclonal, Jackson ImmunoResearch, catalog 711-166-152) in staining buffer. After a final wash, samples were mounted in Vectashield (Vector Labs, catalog H-1000) with $50 \mu \mathrm{g} / \mathrm{mL}$ DAPI. Lung sections were imaged using a $\times 20$ oil immersion objective on an SP5 confocal microscope (Leica).

NK cell isolation and adoptive transfer. NK cells from C57BL/6 mice were adoptively transferred into $\mathrm{Il}_{2 \mathrm{rg}^{-/}} \mathrm{Rag} 2^{-/-}$mice according to previously published protocols (53). Briefly, 8-week-old C57BL/6 mice were euthanized according to institutional protocol. With sterile technique, the spleen was removed and dissociated through a $40-\mu \mathrm{m}$ mesh nylon filter (Fisher Scientific, catalog 22363547) in 0.1\% BSA in sterile saline. The isolated splenocytes were counted using an automated Countess II FL cell counter (Invitrogen) and concentrated to 1 $\times 10^{8}$ cells $/ \mathrm{mL}$. NK cells were isolated from splenocytes via negative magnetic selection (StemCell Technologies, catalog 19855). NK cells $\left(1 \times 10^{6}\right.$ in $200 \mu \mathrm{L}$ sterile PBS $)$ were injected i.p. into $\mathrm{Il} \mathrm{rg}^{-/-} \mathrm{Rag}^{-/-}$ mice and allowed to expand in vivo for 5 days prior to experiments. NK cell expansion was confirmed via flow cytometry. Supplemental Figure 3 compares NK cell phenotypes among WT lung and spleen and 5 days following adoptive transfer.

Human subjects. Seven clinical cases of severe PGD were identified with surgical lung biopsies within the first 30 days following lung transplantation. Six clinical cases of routine lung transplant surveillance bronchoscopies in subjects with no PGD were selected as negative controls. Formalin-fixed and paraffin-embedded samples were recut and stained in separate sections with H\&E, anti-CD56 (clone CD564, Leica Biosystems, catalog PA0192), and anti-CD3 (clone LN10, Leica Biosystems, catalog PA0122). Cells of interest were quantified in 20 high powered fields (HPF, $\times 20$ magnification) for each sample and repeated. Bronchoalveolar lavage fluid (BAL) was prospectively collected and analyzed as part of routine clinical care for subjects who underwent lung transplantation between November 2013 and April 2016. We included all consenting adult subjects who received single lung, bilateral lung, or heart-lung allografts at UCSF and received at least one adequate BAL sample within the first 3 months following transplantation. Samples were considered adequate if at least 50 NK cells were identified by flow cytometry. Our institution performs bronchoscopy with lavage and transbronchial biopsies as part of routine surveillance at 0.5 , 1,2 , and 3 months after transplantation. Additional bronchoscopies are performed when clinically indicated for suspicion of acute infection or rejection. Primary graft dysfunction was graded for all subjects according to international criteria (7). Subject clinical data were obtained by chart review of UCSF records and from the United Network for Organ Sharing (UNOS) database. Standard posttransplant induction regimens 
for all subjects included methylprednisolone, mycophenolate mofetil, and $20 \mathrm{mg}$ i.v. basiliximab intraoperatively and on postoperative day 4 . Initial maintenance immunosuppressant therapy included tacrolimus, prednisone, and mycophenolate mofetil.

Human BAL immunophenotyping. NK cells from fresh BAL samples were phenotyped as described previously $(46,54)$. Each sample was treated with human aggregated immunoglobulins to block nonspecific binding and stained with viability dye (eBioscience, catalog 65-086318). NK cell subsets were identified using: Allophycocyanin-conjugated (APC-conjugated) anti-NKG2A(clone 131411, R\&D Systems, catalog FAB1059A), R-phycoerythrin-conjugated (PE-conjugated) anti-KIR2D (clone NKVFS1, Miltenyi Biotec, catalog 130-092-688), PE-conjugated anti-KIR3D (clone Z27.3.7, Beckman Coulter, catalog IM3292), peridinin-chlorophyll-protein complex-conjugated (PerCP-conjugated) anti-KIR3DL1 (clone DX9, BioLegend, catalog 312718), PE-Cy7-conjugated anti-CD56 (clone HCD56, BioLegend, catalog 318318), Alexa Fluor 700-conjugated anti-CD45 (clone HI30, BioLegend, catalog 304024), and allophycocyanin-cyanine 7-conjugated (APC-Cy7-conjugated) anti-CD3 (clone SK7, BioLegend, cata$\log$ 344818). Samples were acquired with a Beckman Coulter Navios cytometer and data were analyzed with the Kaluza software package (Beckman Coulter). Negative thresholds were set based on no-fluorochrome controls. The gating strategy for human BAL specimens is displayed in Supplemental Figure 8. Viable lymphocytes were identified by viability dye exclusion, $\mathrm{CD} 45^{+}$expression, and forward and side light scatter profiles. NK cells were identified as CD56 ${ }^{+} \mathrm{CD} 3^{-}$.

Statistics. Outcome variable distributions were assessed for normality visually and with the Shapiro-Wilks test. Parametric data were analyzed using a 2-tailed unpaired Student's $t$ test, and 2-tailed Mann-Whitney $U$ tests were applied for nonparametric data. A $P$ value less than 0.05 was considered significant. Comparisons of means in experiments with multiple groups were made with Kruskal-Wallis test, and Dunn's test was used to assess post hoc differences. Comparisons between lung and peripheral blood NK cell phenotypes were made with a paired Student's $t$ test and Pearson's correlation coefficient. Results were visualized using box and whisker plots showing individual data points bound by boxes at 25 th and 75 th percentiles and medians depicted with bisecting lines.

For the human BAL data, the association between NK cells and PGD grade as a dependent ordinal variable on postoperative day 3 was determined using cumulative linked mixed model (CLM) regression analysis to account for repeat measures among individual subjects. Differences between NK cell surface markers in BAL were determined with paired Student's $t$ tests.

Differences between CD16 genotype and PGD as a binary variable were determined with generalized linear models adjusted for transplant characteristics known to cause genetic variation or to impact early outcomes: recipient age, transplant type, transplant indication, sex, and ethnicity. Statistical analyses and visualization were performed in $\mathrm{R}(\mathrm{R}$ Foundation for Statistical Computing) using packages "mgcv," "ordinal," "ggplot," "stringr," "multcomp," "ggbeeswarm," and "ggpubr."

Study approval. The UCSF institutional review board approved the human subject components of this study under protocol 13-10738. Written informed consent was obtained from all participants prior to inclusion in the study. All animal procedures and experiments were conducted according to protocols approved by the UCSF Institutional Animal Care Use Committee.

\section{Author contributions}

DRC, JRG, LLL, MRL, and SRH designed the study. DRC, EA, BM, PW, MN, SJC, FL, and OAA performed experiments. DRC, JRG, OAA, DD, and JPS analyzed results. DRC, JRG, MN, LLL, JAG, and JK provided vital data and data interpretation. DRC and JRG prepared the manuscript with input from all authors.

\section{Acknowledgments}

We thank William Karlon, Chiyo Uchida, Brandon Lim, Ryan Tsiao, Brandon Cowan, and Malori Mindo for instrumental aid in collecting and processing study samples. We also thank Mary Ellen Kleinhenz, Lorianna Leard, Rupal Shah, Nicholas Kolaitis, Aida Venado, and the other members of the clinical lung transplant team for their care of our patients and design of the clinical protocols. We are thankful for the cooperation of Donor Network West, for all of the organ and tissue donors, and for their families for giving gifts of life and knowledge with their generous donations. This project was funded with support from the UCSF Nina Ireland Program for Lung Health (NIPLH) Innovative Grant program (to JRG), Veterans Affairs Office of Research and Development CX002011 (to JRG), the Joel D. Cooper Award from the International Society for Heart and Lung Transplantation (to DRC), the Cystic Fibrosis Foundation Harry Shwachman Career Development Award CALABR19Q0 (to DRC), and NHLBI grants HL151552 (to JRG) and HL130324 (to MRL). LLL is supported in part by the Parker Institute for Cancer Immunotherapy and NIH AI068129 and OAA is a Cancer Research Institute-Irvington Postdoctoral Fellow. We acknowledge the Parnassus Flow Cytometry Core (Research Resource Identifier: SCR 018206) supported in part by NIH grant P30 DK063720 and NIH Instrumentation Grant S10 1S10OD026940-01.

Address correspondence to: Daniel R. Calabrese, San Francisco VA Medical Center, 4150 Clement Street, San Francisco, CA 94121, USA. Phone: 415.221.4810; Email: daniel.calabrese@ucsf.edu.
1. Lund LH, et al. The registry of the international society for heart and lung transplantation: thirty-fourth adult heart transplantation report; focus theme: allograft ischemic time. J Heart Lung Transplant. 2017;36(10):1037-1046.

2. Oto $\mathrm{T}$, et al. Association between primary graft dysfunction among lung, kidney and heart recipients from the same multiorgan donor. Am J Transplant. 2008;8(10):2132-2139.

3. Christie JD, et al. The effect of primary graft dysfunction on survival after lung transplantation.
Am J Respir Crit Care Med. 2005;171(11):1312-1316.

4. Daud SA, et al. Impact of immediate primary lung allograft dysfunction on bronchiolitis obliterans syndrome. Am J Respir Crit Care Med. 2007;175(5):507-513.

5. Siegleman SS, et al. Pulmonary reimplantation response. Ann Surg. 1973;177(1):30-36.

6. Matute-Bello G, et al. Animal models of acute lung injury. Am J Physiol Lung Cell Mol Physiol. 2008;295(3):L379-L399.

7. Snell GI, et al. Report of the ISHLT working group on primary lung graft dysfunction, part I: definition and grading-A 2016 consensus group statement of the international society for heart and lung transplantation. J Heart Lung Transplant. 2017;36(10):1097-1103.

8. Sayah DM, et al. Neutrophil extracellular traps are pathogenic in primary graft dysfunction after lung transplantation. Am J Respir Crit Care Med. 2015;191(4):455-463.

9. den Hengst WA, et al. Lung ischemia-reperfusion injury: a molecular and clinical view on a complex 
pathophysiological process. Am J Physiol Heart Circ Physiol. 2010;299(5):H1283-1299.

10. Cooper GE, et al. Human $\mathrm{CD} 49 \mathrm{a}^{+}$lung natural killer cell cytotoxicity in response to influenza a virus. Front Immunol. 2018;9:1671.

11. Vivier $\mathrm{E}$, et al. Innate or adaptive immunity? The example of natural killer cells. Science. 2011;331(6013):44-49.

12. Raulet DH, et al. Regulation of ligands for the NKG2D activating receptor. Annu Rev Immunol. 2013;31:413-441.

13. Feng $L$, et al. The effect of renal ischemia-reperfusion injury on expression of RAE- 1 and $\mathrm{H} 60$ in mice kidney. Transplant Proc. 2006;38(7):2195-2198.

14. Zhang Z-X, et al. NK cells induce apoptosis in tubular epithelial cells and contribute to renal ischemia-reperfusion injury. JImmunol. 2008;181(11):7489-7498.

15. Zingoni A, et al. Natural killer cell response to chemotherapy-stressed cancer cells: role in tumor immunosurveillance. Front Immunol. 2017;8:1194.

16. Chen GE, et al. Toll-like receptor 4 engagement contributes to expression of NKG2D ligands by renal tubular epithelial cells. Nephrol Dial Transplant. 2011;26(12):3873-3881.

17. Victorino F, et al. Tissue-resident NK cells mediate ischemic kidney injury and are not depleted by anti-asialo-GM1 antibody. J Immunol. 2015;195(10):4973-4985.

18. Abel AM, et al. Natural killer cells: development, maturation, and clinical utilization. Front Immunol. 2018;9(1869).

19. Alter G, et al. CD107a as a functional marker for the identification of natural killer cell activity. JImmunol Methods. 2004;294(1-2):15-22.

20. Aquino-Galvez A, et al. MICA polymorphisms and decreased expression of the MICA receptor NKG2D contribute to idiopathic pulmonary fibrosis susceptibility. Hum Genet. 2009;125(5-6):639-648.

21. Wesselkamper SC, et al. NKG2D is critical for NK cell activation in host defense against pseudomonas aeruginosa respiratory infection. J Immunol. 2008;181(8):5481-5489.

22. Borchers MT, et al. Sustained CTL activation by murine pulmonary epithelial cells promotes the development of COPD-like disease. JClin Invest. 2009;119(3):636-649.

23. Barrow $A D$, et al. The natural cytotoxicity receptors in health and disease. Front Immunol. 2019;10:909.

24. Vadstrup K, Bendtsen F. Anti-NKG2D mAb: a new treatment for Crohn's disease? Int J Mol Sci. 2017;18(9):1997.

25. Molfetta R, et al. Regulation of NKG2D-dependent
NK cell functions: the yin and the yang of receptor endocytosis. Int JMol Sci. 2017;18(8):1677.

26. Guerra N, et al. NKG2D-deficient mice are defective in tumor surveillance in models of spontaneous malignancy. Immunity. 2008;28(4):571-580.

27. Wang $\mathrm{H}$, et al. The unexpected effect of cyclosporin A on CD56+CD16- and CD56+CD16+ natural killer cell subpopulations. Blood. 2007;110(5):1530-1539.

28. Wasik M, et al. Effect of FK506 versus cyclosporine on human natural and antibody-dependent cytotoxicity reactions in vitro. Transplantation. 1991;51(1):268-270.

29. Martin JF, et al. An IL-2 paradox: blocking CD25 on T cells induces IL-2-driven activation of CD56(bright) NK cells. J Immunol. 2010;185(2):1311-1320.

30. Lehmann MH, et al. CCL2 expression is mediated by type I IFN receptor and recruits NK and T cells to the lung during MVA infection. J Leukoc Biol. 2016;99(6):1057-1064.

31. Champsaur M, Lanier LL. Effect of NKG2D ligand expression on host immune responses. Immunol Rev. 2010;235(1):267-285.

32. Frick AE, et al. Early protein expression profile in bronchoalveolar lavage fluid and clinical outcomes in primary graft dysfunction after lung transplantation. Eur J Cardiothorac Surg. 2020;58(2):379-388.

33. Hoffman SA, et al. Plasma cytokines and chemokines in primary graft dysfunction post-lung transplantation. Am J Transplant. 2009;9(2):389-396.

34. Li F, et al. Natural killer cells are involved in acute lung immune injury caused by respiratory syncytial virus infection. J Virol. 2012;86(4):2251-2258.

35 . Hoegl S, et al. NK cells regulate CXCR2 $2^{+}$neutrophil recruitment during acute lung injury. J Leukoc Biol. 2017;101(2):471-480.

36. Bharat A, et al. Antibodies to self-antigens predispose to primary lung allograft dysfunction and chronic rejection. Ann Thorac Surg. 2010;90(4):1094-1101.

37. Shah RJ, et al. Plasma monocyte chemotactic protein-1 levels at 24 hours are a biomarker of primary graft dysfunction after lung transplantation. Transl Res. 2012;160(6):435-442.

38. Mallavia B, et al. Mitochondrial DNA stimulates TLR9-dependent neutrophil extracellular trap formation in primary graft dysfunction. Am J Respir Cell Mol Biol. 2020;62(3):364-372.

39. Wu J, Lanier LL. Natural killer cells and cancer. Adv Cancer Res. 2003;90:127-156.

40. Bharat A, et al. Lung-restricted antibodies medi- ate primary graft dysfunction and prevent allotolerance after murine lung transplantation. Am J Respir Cell Mol Biol. 2016;55(4):532-541.

41. Kaza V, et al. Pre-existing self-reactive IgA antibodies associated with primary graft dysfunction after lung transplantation. Transpl Immunol. 2020:101271.

42. Bharat A, Kreisel D. Immunopathogenesis of primary graft dysfunction after lung transplantation. Ann Thorac Surg. 2018;105(3):671-674.

43. Verrier $\mathrm{T}$, et al. Phenotypic and functional plasticity of murine intestinal NKp46+ group 3 innate lymphoid cells. JImmunol. 2016;196(11):4731-4738.

44. Monticelli LA, et al. Lung innate lymphoid cell composition is altered in primary graft dysfunction. Am J Respir Crit Care Med. 2020;201(1):63-72.

45. Calabrese DR, et al. Natural killer cells in lung transplantation. Thorax. 2019;74(4):397-404.

46. Calabrese DR, et al. NKG2C natural killer cells in bronchoalveolar lavage are associated with cytomegalovirus viremia poor outcomes in lung allograft recipients. Transplantation. 2019;103(3):493-501.

47. Greenland JR, et al. HLA mismatching favoring host-versus-graft NK cell activity via KIR3DL1 is associated with improved outcomes following lung transplantation. Am J Transplant. 2017;17(8):2192-2199.

48. Okazaki M, et al. A mouse model of orthotopic vascularized aerated lung transplantation. Am J Transplant. 2007;7(6):1672-1679.

49. Karnbach C, et al. Immune rejection of a large sarcoma following cyclophosphamide and IL-12 treatment requires both NK and NK $\mathrm{T}$ cells and is associated with the induction of a novel NK T cell population. J Immunol. 2001;167(5):2569-2576.

50. Jamin C, et al. Multi-center harmonization of flow cytometers in the context of the European "PRECISESADS" project. Autoimmun Rev. 2016;15(11):1038-1045

51. Ruscher R, Hogquist KA. Intravenous labeling and analysis of the content of thymic perivascular spaces. Bio Protoc. 2018;8(5):e2757.

52. Cleary SJ, et al. Complement activation on endothelium initiates antibody-mediated acute lung injury. JClin Invest. 2020;130(11):5909-5923.

53. Min-Oo G, Lanier LL. Cytomegalovirus generates long-lived antigen-specific NK cells with diminished bystander activation to heterologous infection. J Exp Med.2014;211(13):2669-2680.

54. Greenland JR, et al. Bronchoalveolar lavage cell immunophenotyping facilitates diagnosis of lung allograft rejection. Am J Transplant. 2014;14(4):831-840. 